\title{
Genetic dissection of photosynthetic efficiency traits for enhancing seed yield in chickpea
}

\author{
Udita Basu $^{1}$ | Deepak Bajaj ${ }^{1}$ | Akash Sharma $^{1}$ | Naveen Malik ${ }^{1}$ | Anurag Daware ${ }^{1}$ | \\ Laxmi Narnoliya ${ }^{1}$ | Virevol Thakro ${ }^{1}$ | Hari D. Upadhyaya ${ }^{2}$ | Rajendra Kumar ${ }^{3}$ | \\ Shailesh Tripathi ${ }^{4}$ | Chellapilla Bharadwaj ${ }^{4}$ | Akhilesh K. Tyagi ${ }^{1}$ | Swarup K. Parida ${ }^{1}$
}

\footnotetext{
${ }^{1}$ National Institute of Plant Genome Research (NIPGR), Aruna Asaf Ali Marg, New Delhi 110067, India

${ }^{2}$ International Crops Research Institute for the Semi-Arid Tropics (ICRISAT), Patancheru 502324, India

${ }^{3}$ U.P. Council of Agricultural Research, Gomati Nagar, Lucknow 226010, India

${ }^{4}$ Division of Genetics, Indian Agricultural Research Institute (IARI), New Delhi 110012, India

Correspondence

S. K. Parida, National Institute of Plant Genome Research (NIPGR), Aruna Asaf Ali

Marg, New Delhi 110067, India.

Email: swarup@nipgr.ac.in; swarupdbt@gmail. com

Present Address

Akhilesh K. Tyagi, Department of Plant

Molecular Biology, University of Delhi, South

Campus, New Delhi 110021, India

Funding information

Department of Biotechnology (DBT), Ministry of Science and Technology
}

\begin{abstract}
Understanding the genetic basis of photosynthetic efficiency (PE) contributing to enhanced seed yield per plant (SYP) is vital for genomics-assisted crop improvement of chickpea. The current study employed an integrated genomic strategy involving photosynthesis pathway gene-based association mapping, genome-wide association study, quantitative trait loci (QTL) mapping, and expression profiling. This identified 16 potential single nucleotide polymorphism loci linked to major QTLs underlying 16 candidate genes significantly associated with PE and SYP traits in chickpea. The allelic variants were tightly linked to positively interacting QTLs regulating both enhanced PE and SYP traits as exemplified by a chlorophyll A-B binding protein-coding gene. The leaf tissue-specific pronounced up-regulated expression of 16 associated genes in germplasm accessions and homozygous individuals of mapping population was evident. Such combinatorial genomic strategy coupled with gene haplotype-specific association and in silico protein-protein interaction study delineated natural alleles and superior haplotypes from a chlorophyll A-B binding (CAB) protein-coding gene and its interacting gene, Timing of CAB Expression 1 (TOC1), which appear to be most promising candidates in modulating chickpea PE and SYP traits. These functionally pertinent molecular signatures identified have efficacy to drive marker-assisted selection for developing PE-enriched cultivars with high seed yield in chickpea.
\end{abstract}

\section{KEYWORDS}

chickpea, GWAS, photosynthesis, QTL, SNP, yield

\section{1 | INTRODUCTION}

In order to sustain global food security, it is imperative to enhance the yield and productivity of crop plants, especially of the staple food crops such as major cereals and legumes. The food we consume is the resultant of millions of years of evolution which provided the green plants the ability to fix the atmospheric carbon dioxide into carbohydrate using the solar energy. The photosynthetic carbon metabolism (PCM) is thus considered as one of the major contributor to crop grain yield. Since long time, researchers all over the world have made substantial efforts to manipulate the metabolic enzymes controlling photosynthesis in order to enhance the crop yield and productivity. The ribulose-1,5-bisphosphate carboxylase (RuBisCO) is considered as one of the major target of this manipulation, and by engineering of this metabolic enzyme, photosynthetic carbon fixation gain could increase by $30 \%$ in the environment (Zhu, Ort, Whitmarsh, \& Long, 2004). Therefore, a better understanding on regulation of photosynthesis metabolism at a global scale using advanced structural, functional, and comparative genomics as well as molecular genetics strategies is essential to accomplish the prime objective of crop yield enhancement. In this perspective, a PCM-associated HIGHER YIELD RICE transcription factor enhancing photosynthesis and subsequent 
grain yield during normal and stress (drought and high-temperature) conditions is found much promising in rice (Ambavaram et al., 2014). Moreover, the manipulation of cytochrome $b_{6} / f$ complex in the electron transport chain appears to be a potential target for enhancing photosynthetic rates and yield in crop plants (De Souza et al., 2017; Yamori et al., 2016). The aforesaid studies collectively highlighted that photosynthesis is the most vital and basic metabolic pathway essential for energy generation and survival and overall growth and development of crop plants. Henceforth, the PCM and major traits contributing to high photosynthetic efficiency (PE) need to be optimized at a global scale employing diverse genomics-assisted breeding and functional genomic approaches for the increase in crop yield and productivity. Recently, a genome-wide association study (GWAS) of diverse chlorophyll traits including total chlorophyll content is found efficient to identify genomic loci (genes) governing PCM for genetic improvement of legumes including soybean (Dhanapal et al., 2016). Unfortunately, no such information pertaining to PCM regulation is available in chickpea that necessitates comprehensive understanding on complex genetic architecture of vital traits contributing to high PCM and $\mathrm{PE}$ as well as increased seed yield in this legume crop.

Chickpea (Cicer arietinum), a vital food legume crop, is primarily represented by two of its domesticated desi and kabuli cultivars exhibiting distinct agro-morphological characteristics. Numerous genetic resources in form of germplasm accessions, landraces, cultivated varieties, genetic stocks, and wild species accessions as well as advanced generation mapping population contrasting for diverse agronomic traits are accessible in chickpea at various international and national Genebank. The draft genome sequences of desi and kabuli and its progenitor wild Cicer reticulatum accession are decoded recently in chickpea (Gupta et al., 2016; Jain et al., 2013; Parween et al., 2015; Varshney, Song, et al., 2013). Moreover, the transcriptome and genome resequencing of multiple cultivated (desi and kabuli) and wild chickpea accessions generated a diverse array of genes, transcription factors, and regulatory sequences as well as simple sequence repeat and single nucleotide polymorphism (SNP) markers at a genome-wide scale (Pandey et al., 2016; Varshney, Mohan, et al., 2013). The available sequence information is also useful in deciphering the structural and functional perspectives of protein coding genes controlling diverse known regulatory pathways/networks especially underlying the basic metabolic processes such as photosynthesis at a whole genome level in crop plants (http://www genome.jp/kegg/pathway.html).

Development of high-yielding climate resilient cultivars in chickpea is the prime focus worldwide. Increasing photosynthetic carbon fixation rate comes up as an attractive strategy for further yield enhancement in this vital legume food crop. This requires a comprehensive understanding of the photosynthetic gene regulatory pathways/networks and manipulation of gene(s) involved to achieve high $\mathrm{PE}$ and increased seed yield. The rapid genetic dissection of complex PE traits controlling increased seed yield is now certainly feasible through deployment of an integrated genomics-assisted breeding strategy involving association mapping, quantitative trait loci (QTL) mapping, expression profiling, and molecular haplotyping in chickpea (Kale et al., 2015; Kujur et al., 2015, 2015b, 2016). The combinatorial genomic strategy will be useful to scan functionally pertinent novel molecular tags including potential genes and superior alleles influencing PE for enhancing yield in chickpea.

In these perspectives, the present study employed high-resolution gene-based association analysis and GWAS integrated with QTL mapping and transcript profiling in phenotypically well-characterized natural germplasm accessions and mapping population to scan the most promising molecular signatures (SNP marker, genes/QTLs and natural alleles) regulating high PE vis-à-vis increased seed yield per plant (SYP) in chickpea.

\section{2 | MATERIALS AND METHODS}

\subsection{Genetic resource and phenotyping}

An association panel of 92 accessions including 38 desi and 54 kabuli core, minicore, and reference core germplasm accessions representing diverse ecogeographical locations of 20 countries of the world was constituted in accordance with Kujur et al. (2015a; 2015b; Table S1). These were grown in the experimental field during normal crop season (October to February) as per randomized complete block design and/ or alpha-lattice design with two replications following the standard agronomic practices. To develop an intraspecific mapping population contrasting with PE traits, two accessions, ICC 4958 (desi landrace originated from central India) and ICC 12299 (desi landrace from Nepal) exhibiting high and low PE, respectively, were selected from our aforesaid constituted association panel of chickpea. Subsequently, an intraspecific $\mathrm{F}_{9}$ recombinant inbred lines (RIL) mapping population (ICC $4958 \times$ ICC 12299) composing of 236 individuals was grown in field according to aforesaid strategies.

These natural germplasm accessions and mapping population were phenotyped for diverse PE and SYP traits for successive years (2013 and 2014) at International Crops Research Institute for the Semi-Arid Tropics, Hyderabad (latitude $17^{\circ} 3^{\prime} \mathrm{N} /$ longitude $77^{\circ} 2^{\prime} \mathrm{E}$ ). For determining $\mathrm{PE}$, diverse major traits including total chlorophyll content, chlorophyll fluorescence, and $\mathrm{CO}_{2}$ assimilation rate were measured. To estimate chlorophyll content (CC), the total chlorophyll was extracted from the liquid nitrogen-grounded homogenate of $10-$ 15 days old seedlings (fresh weight of $0.5 \mathrm{~g}$ ) with $2 \mathrm{ml}$ chilled $80 \%$ acetone, and their concentration were measured ( $\mathrm{mg} / \mathrm{g}$ fresh weight) by an ultraviolet-visible spectrophotometer at 663,645 , and $470 \mathrm{~nm}$ following the methods of S. C. Saxena et al. (2013). For precise estimation of chlorophyll content, third and fourth leaves (representing the plant canopy) of 30-40 days after sowing (DAS) grown plants were considered to be the most suitable leaf positions for chlorophyll measurement in chickpea. These leaf tissues were used for SPAD chlorophyll meter reading (SCMR) by SPAD-502 Plus meter (KONICA MINOLTA CO. LTD., JAPAN) as per Kashiwagi, Krishna, Singh, and Upadhyaya (2006). The chlorophyll fluorescence (CF) and $\mathrm{CO}_{2}$ gas exchange rate were measured from the third and fourth attached leaves of 60-90 DAS old plants by estimating the maximal quantum yield of PSII (Fv/Fm) employing the modulated chlorophyll fluorometer and LI-6400XT (LI-COR Inc., USA) as per Ambavaram et al. (2014). Especially, the mean $\mathrm{CO}_{2}$ exchange rate was estimated by selecting three to five representative plants, after $6 \mathrm{hr}$ of illumination 
with a daily continuous photoperiodic cycle of 10-hr light and 14-hr dark at the $22-24{ }^{\circ} \mathrm{C}$ leaf temperature, $400-500 \mu \mathrm{mol} / \mathrm{s} \mathrm{CO}_{2}$ and $65-70 \%$ relative humidity. To determine the $\mathrm{CO}_{2}$ exchange rate precisely, $\mathrm{CO}_{2}$ assimilation rate at increasing $\mathrm{CO}_{2}$ concentration $\left(\mathrm{CAR} \uparrow \mathrm{CO}_{2}\left[\mu \mathrm{mol} \cdot \mathrm{CO}_{2} \cdot \mathrm{m}^{-2} \cdot \mathrm{s}^{-1}\right]\right)$ and $\mathrm{CO}_{2}$ assimilation rate at increasing light intensity (CAR个LI $\left.\left[\mu \mathrm{mol} \cdot \mathrm{CO}_{2} \cdot \mathrm{m}^{-2} \cdot \mathrm{s}^{-1}\right]\right)$ were measured following Ambavaram et al. (2014). Three biological replicates, each with three technical replicates, were used for measuring the individual target traits in accessions and RIL mapping individuals of chickpea. SYP was measured by weighing the mean weight (g) of fully matured dried seeds ( $10 \%$ moisture content) harvested from 5 to 10 representative plants of each germplasm accession and RIL mapping individual. The genetic inheritance pattern of all these studied traits using diverse statistical parameters including coefficient of variation (CV), broadsense heritability $\left(\mathrm{H}^{2}\right)$, Pearson's correlation coefficient $(r)$, and frequency distribution was determined in an association panel and a RIL mapping population as per the methods described earlier (Bajaj, Upadhyaya et al., 2015). CV was determined by estimating the ratio of standard deviation to the mean for each individual environment. We measured the significant effect between gene/genotype and environment by estimating the genotypes (G; accessions/mapping individuals) and phenotyping experimental years/environments (E) interaction $(\mathrm{G} \times \mathrm{E})$ using analysis of variance as per Upadhyaya et al. (2016). The analysis of variance outcomes were further used to estimate the broad-sense heritability $\left.\sigma^{2} g\left(\sigma^{2} g+\sigma^{2} g e n+\sigma^{2} e n r\right)\right]$ following $\sigma^{2}$ g (genetic), $\sigma^{2}$ ge $\left(\mathrm{G} \times \mathrm{E}\right.$ ) and $\sigma^{2}$ e (error) variance with $n$ (number of experimental years/environments) $=2$ and $r$ (number of replicates) $=2$

\section{2 | Targeted gene amplicon resequencing-based SNP mining and genotyping}

A selected set of candidate genes annotated from kabuli genome and reported to be involved in photosynthesis-related metabolic pathways (cam00195, cam00196, and cam00710) in chickpea was retrieved from Kyoto Encyclopedia of Genes and Genomes (KEGG) database (http://www.genome.jp/kegg/pathway.html). The coding DNA sequences (CDS) of these genes ( $\mathrm{NCBI} \mathrm{C}$. arietinum Annotation Release 101, https://www.ncbi.nlm.nih.gov/genome/annotation_euk/ Cicer_arietinum/101/\%23BuildInfo) were BLASTN searched against the previously released CDS of kabuli genes (Varshney, Mohan, et al., 2013; http://gigadb.org/dataset/100076) to obtain the best possible true gene homologues ( $E$ : 0 with $\geq 500$ bit score) of chickpea. This exertion was performed to synchronize the version of reference kabuli genome-led gene annotation information utilized in the aforesaid candidate gene-based SNP analysis with that of whole genome genotyping-by-sequencing (GBS)-derived SNP genotyping data available with us for GWAS of PE and SYP traits in chickpea.

The identified chickpea genes were further resequenced using the genomic DNA of 92 diverse desi and kabuli germplasm accessions (association panel) of chickpea employing the multiplexed amplicon resequencing strategy (TruSeq Custom Amplicon v1.5) of Illumina MiSeq next-generation sequencer (Illumina, USA). The $2 \mathrm{~kb}$ upstream regulatory regions (URRs), exons/CDS, introns, and $2 \mathrm{~kb}$ downstream regulatory regions (DRRs) of these genes were selected for designing and synthesizing the custom oligo-probes (producing amplicons with mean size of 500 BP per reaction) using Illumina Design Studio. The probe-pooling, template libraries-constitution, sample-specific indices addition to individual libraries and normalization of uniquely tagged pooled amplicon libraries were performed as per M. S. Saxena, Bajaj, Das, et al. (2014) and Malik et al. (2016). Accordingly, the sequencing of generated clusters by Illumina MiSeq platform, mapping of sequenced gene amplicons of each chickpea accession using the pseudomolecules of kabuli chickpea genome (http://gigadb.org/ dataset/100076), and discovery of high-quality SNPs among accessions were carried out as per M. S. Saxena, Bajaj, Das, et al. (2014) and Kujur et al. (2015, 2015a, 2015b).

\section{3 | Trait association mapping}

For association mapping, the genotyping and comprehensive annotation information of candidate gene-derived SNPs and genome-wide SNPs discovered from the resequenced 92 desi and kabuli chickpea accessions (association panel) employing a GBS assay were obtained (Kujur et al., 2015, 2015a, 2015b). Subsequently, the population structure, principal component analysis (PCA), and linkage disequilibrium (LD) decay measured among accessions were acquired from the previous study of Kujur et al. (2015a, 2015b). The accessions revealing high $\mathrm{H}^{2}$ for the studied PE and SYP traits across two diverse environments were acquired for association study. Consequently, environment-wise phenotyping information of these said traits measured from the individual selected accession were utilized to calculate its average trait value for association study. The SNP genotyping data were integrated with multienvironment field phenotyping information of PE and SYP traits as well as kinship $(K)$, population structure $(\mathrm{Q})$, and $\mathrm{PCA}(\mathrm{P})$ information of accessions (association panel). This was performed by mixed model $(\mathrm{P}+\mathrm{K}, \mathrm{K}$ and $\mathrm{Q}+\mathrm{K})$-led compressed mixed linear model (CMLM) and P3D (Kang et al., 2010; Z. Zhang et al., 2010)/efficient mixed model association expedited (EMMAX) strategies of genome association and prediction integrated tool (GAPIT) (Lipka et al., 2012). The reliability of SNP marker-trait association was ascertained by using the quantile-quantile (Q-Q) plot-based false discover rate (FDR) (cut-off $\leq 0.05$, Benjamini \& Hochberg, 1995) correction for multiple comparison between observed/expected $-\log _{10}(p)$ value and adjusted $p$ value threshold of significance, measured in each trait-associated genomic locus. The genomic SNP loci associated with PE and SYP traits at a lowest FDR adjusted $p$ value (cut-off $p<1 \times 10^{-8}$ ) and highest $R^{2}$ were considered significant.

\section{4 | QTL mapping}

The GBS-derived high-quality SNPs (differentiating 92 accessions) showing polymorphism between two parental accessions (ICC 4958 and ICC 12299) were genotyped using the genomic DNA of 236 mapping individuals from a RIL population (ICC 4958 × ICC 12299) through Sequenom MALDI-TOF MassARRAY assay (http://www. sequenom.com) as per M. S. Saxena, Bajaj, Das, et al. (2014) and M. S. Saxena, Bajaj, Kujur et al. (2014). The significant SNP genotyping data were analysed by JoinMap 4.1 (www.kyazma.nl/index.php/mc. JoinMap) with Kosambi mapping function and using high logarithm 


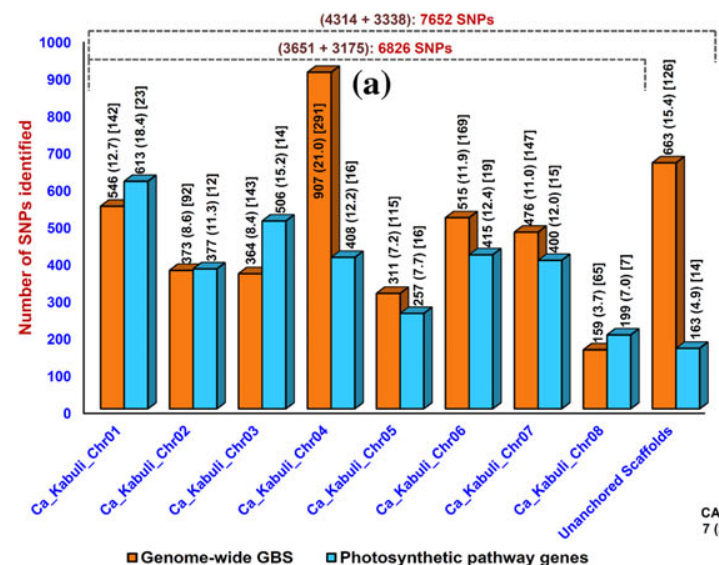

(b)

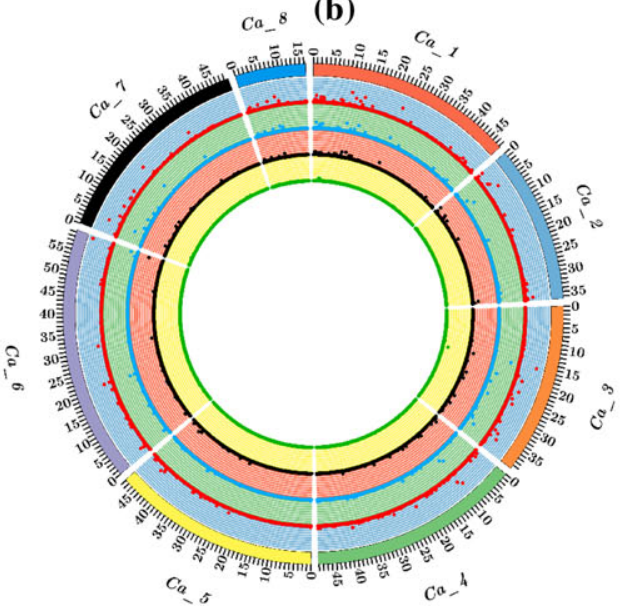

(d)

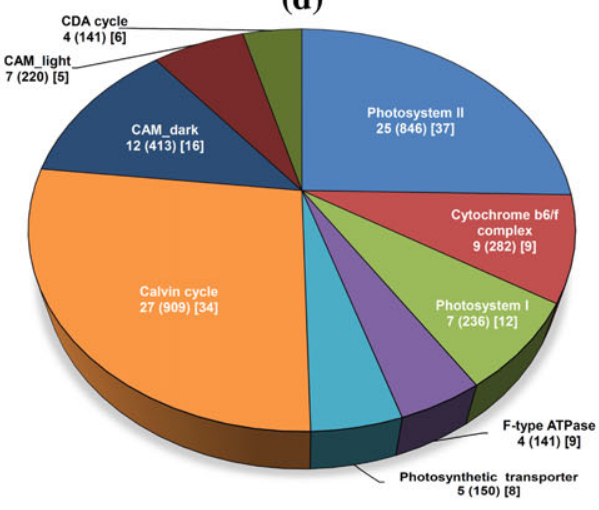

(c)

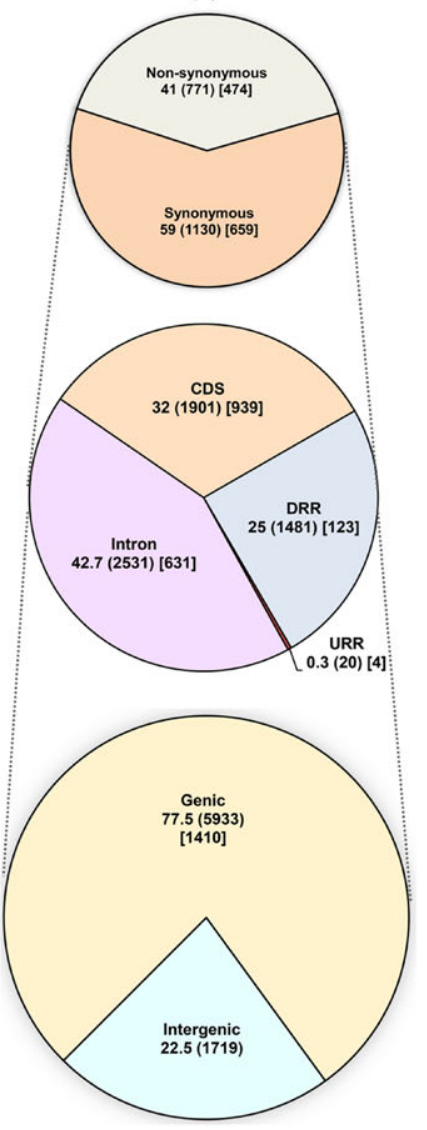

FIGURE 1 Structural and functional annotation of 7,652 single nucleotide polymorphisms (SNPs) including 4,314 genome-wide and 3,338 photosynthesis metabolism pathway gene-based SNPs in chickpea. (a) Frequency distribution of 7,652 SNPs mapped on chromosomes and unanchored scaffolds of kabuli chickpea genome. Numbers within the round and square parentheses above the bars of histogram denote the percentage of SNPs mined and number of genes with SNPs, respectively. Digits within the round parentheses above the dotted lines indicate the total SNPs mapped on chromosomes and unanchored scaffolds. (b) A Circos circular ideogram depicting the genomic distribution of 6,826 SNPs (represented by orange colour dotted circle) mapped on eight kabuli chromosomes. Blue, black, and green colour dotted circles denote the genebased SNPs, nonsynonymous + regulatory SNPs, and nonsynonymous SNPs, respectively. The outermost circles illustrate eight chromosomes denoted with diverse colours. (c) Proportionate distribution of 7,652 SNPs in coding (synonymous and nonsynonymous) and noncoding (intron, URR, and DRR) regions of genes annotated from kabuli genome. CDS = coding DNA sequence; URR/DRR = upstream/downstream regulatory region. (d) Classification of genes with SNPs representing diverse functional modules of photosynthesis KEGG metabolism pathways in chickpea. Digits within the round and square parentheses inside the slices of Pi chart denote the number of SNPs and number of genes with SNPs, respectively. GBS = genotyping-by-sequencing; CAM = crassulacean acid metabolism; CDA = C4 dicarboxylic acid [Colour figure can be viewed at wileyonlinelibrary.com]

of odds (LOD) threshold $(\geq 5.0)$ to estimate the linkages among the SNPs. A high-resolution intraspecific genetic linkage map was constructed by assigning the SNPs into defined linkage groups (LGs; designated as LG1 to LG8)/chromosomes in accordance with their centiMorgan (cM) genetic distances and respective marker physical positions (bp) on chromosomes and further visualized by MapChart v2.2 (Voorrips, 2002).

For molecular mapping of major QTLs governing PE and SYP traits, the genotyping information of SNPs genetically mapped on a high-density linkage map (with eight chromosomes/LGs) was correlated with multienvironment PE and SYP trait field phenotypic data of RIL mapping individuals and parents using a composite interval mapping (CIM) function of MapQTL 6 (Van Ooijen, 2009). For QTL mapping, the LOD cutoff score $>5.0$ with 1,000 permutation at a $p<.05$ was considered most significant in CIM. Accordingly, the phenotypic variation explained
(PVE) and additive effect specified by each major QTL on PE and SYP traits at a significant LOD were determined. The main-effect QTLs (MQTLs) were identified using a CIM function of MapQTL 6 and QTLNetwork v2.0 (http://ibi.zju.edu.cn/software/qtInetwork). The MQTLs and the QTLs involved in epistatic $(Q \times Q)$ and $Q T L s$ by environment $(\mathrm{Q} \times \mathrm{E})$ interactions as defined as epistatic QTLs (E-QTLs) were identified using the three-loci QTL interface of Genotype Matrix Mapping program v.2.1 (www.kajusa.or.jp/GMM). The detail M-QTL and E-QTL mapping strategy followed was adopted from Gautami et al. (2012) and Varshney et al. (2014).

\section{5 | Differential expression profiling}

RNA was isolated from vegetative and reproductive tissues (shoot, root, young/mature leaf [third/fourth leaves of 30-40 DAS grown 


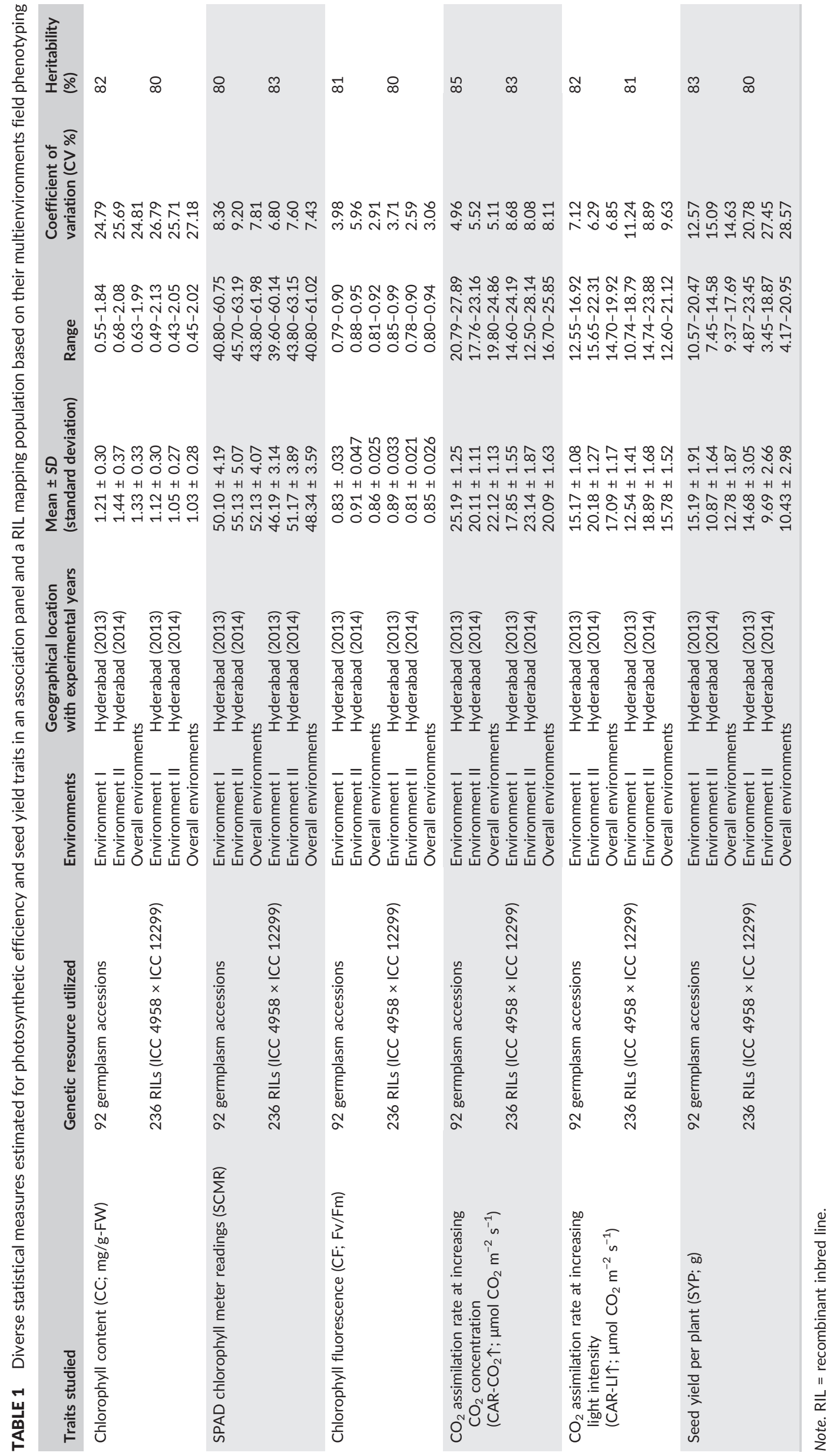




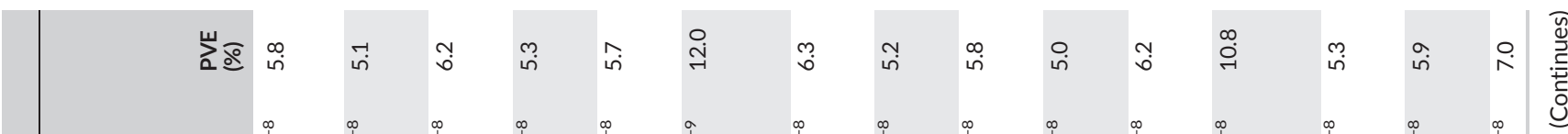

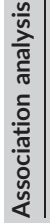

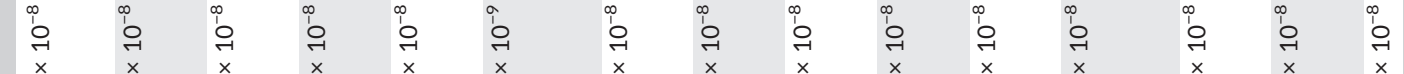

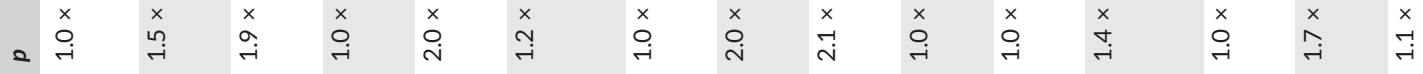

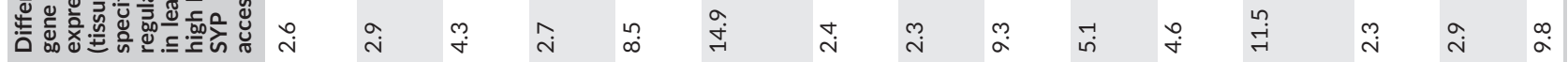

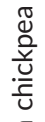

$\frac{\sqrt{\pi}}{\sqrt[n]{2}}$

is

蒙

¿

$\frac{\overline{0}}{\frac{0}{\lambda}}$

्ֶष

몽

崩

ปे

㦶

萢

옹

흘

黄

$\frac{\pi}{8}$

음

崩

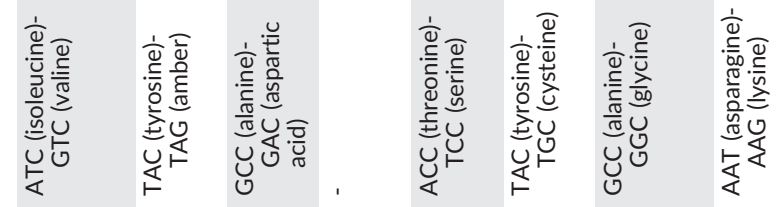

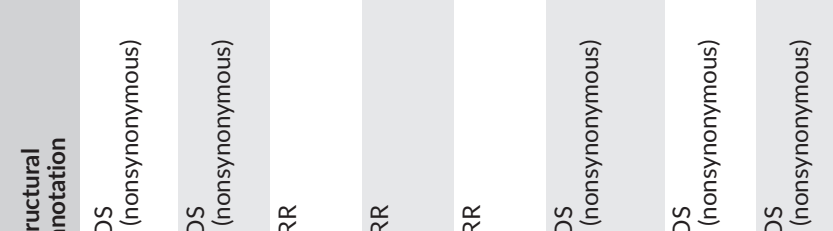
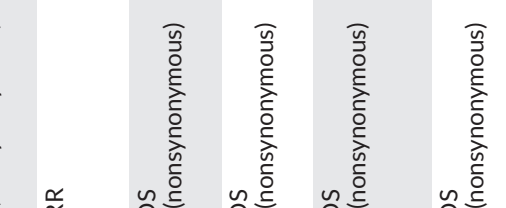

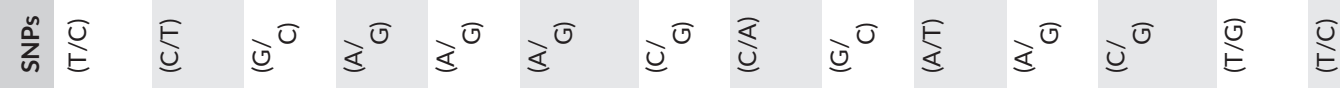

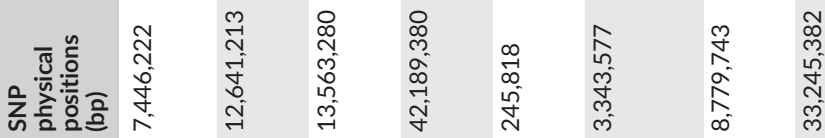

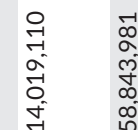

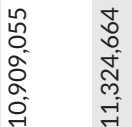

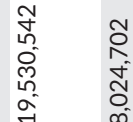

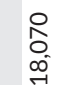

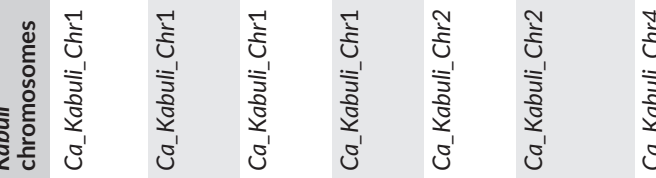

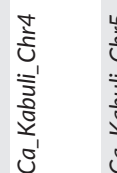

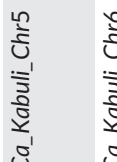

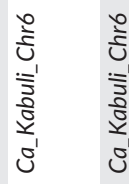

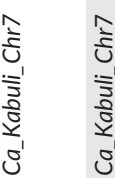

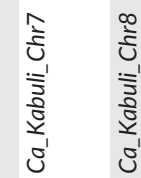

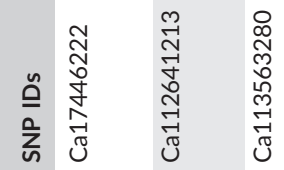

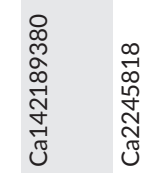

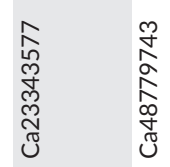

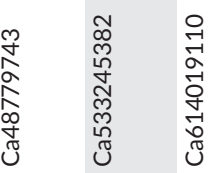

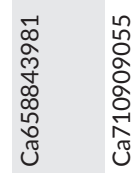

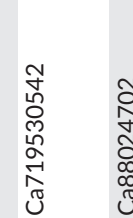

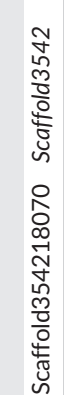

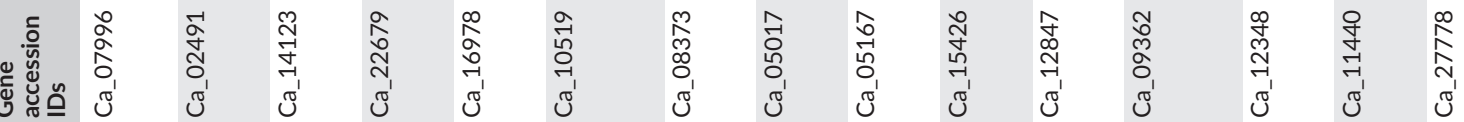

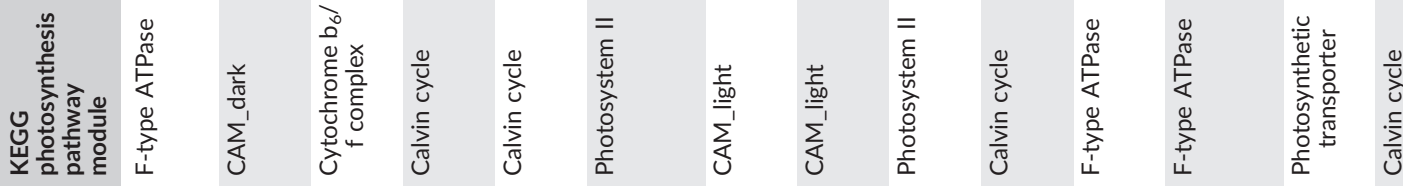

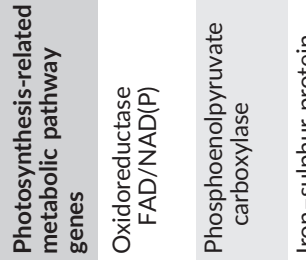
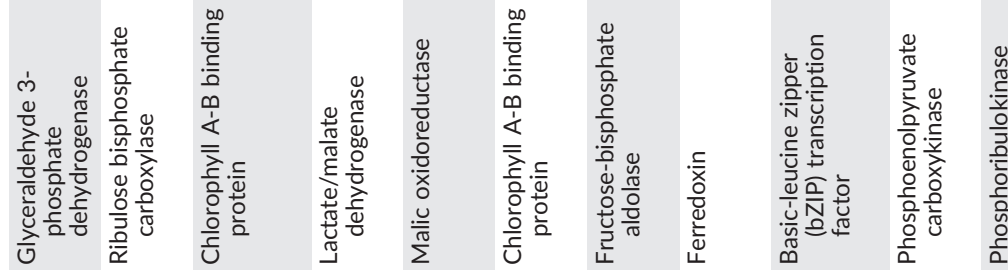
plants], flower bud, young pod, and seed) of parental accessions (ICC 4958 and ICC 12299) and two of each homozygous mapping individuals derived from a RIL population (ICC $4958 \times$ ICC 12299) as well as from eight desi and kabuli accessions (selected from association panel) contrasting with PE and SYP traits. The differential expression analysis was performed by assaying the gene-specific primers among tissues of accessions/individuals as per Bajaj, Saxena, et al. (2015) and Upadhyaya et al. (2015). Briefly, $1 \mu \mathrm{g}$ of high-quality RNA isolated from tissues was utilized to synthesize cDNA by Applied Biosystems (ABI, USA) cDNA synthesis kit. The diluted cDNA and 2X Fast SYBR Green Master Mix (ABI) and 200 nM of forward and reverse gene-based primers were amplified in ABI7500 Fast real-time polymerase chain reaction (RT-PCR) system. Biological and technical replicates aside an internal control gene, elongation factor 1-alpha, were utilized for expression profiling in RT-PCR assay as recommended by Bajaj, Saxena, et al. (2015). The cycle threshold (Ct) expression values obtained for various genes were first normalized with that of the reference elongation factor 1-alpha gene $(\Delta \mathrm{Ct})$. The final values for fold change in expression were derived by calculating $2^{-\Delta \mathrm{Ct}}$ which represents the relative expression level of the gene with respect to the reference gene in that sample assayed. The fold change was calculated for the genes with respect to leaf tissue in high as compared with low PE/SYP accessions and RIL individuals. Significant difference in gene expression was, estimated and differential expression profiles were visualized with a heat map by MultiExperiment Viewer (http://www.tm4.org/mev).

\subsection{In silico protein-protein interaction study}

To scan the known/candidate protein-protein interactions based on experimental data, computational prediction and public literature survey, the amino acid sequence encoded by an Arabidopsis homologue of a strong PE, and SYP trait-associated chickpea gene were analysed in STRING (https://string-db.org) and THALEMINE interface of ARAPORT (https://www.araport.org). The proteins of Arabidopsis genes possibly exhibiting interactions with the proteins of said trait-associated genes were sequence homology (BLAST) searched against the annotated gene protein sequences of kabuli genome (http://gigadb.org/dataset/100076) to identify the true chickpea gene homologues for further analysis.

\section{7 | Trait association potential of interacting genes}

The entire CDS, intron, and $2 \mathrm{~kb}$ of each URR and DRR sequence components of chickpea genes demonstrating possible protein-protein interactions with a strong PE and SYP trait-associated gene were sequenced in 92 desi and kabuli germplasm accessions (association panel) and 236 mapping individuals and parents of a RIL population (ICC $4958 \times$ ICC 12299) using the multiplexed amplicon resequencing strategy of Illumina MiSeq next-generation sequencer to discover the high-quality SNPs as per M. S. Saxena, Bajaj, Das, et al. (2014) and Malik et al. (2016). These gene-derived SNP genotyping information generated from association panel and mapping population were used for high-resolution association and QTL mapping respectively following aforesaid strategy. For molecular haplotyping and gene haplotype-specific trait association and expression analysis, the constitution of SNP-haplotypes and high-resolution LD mapping in the genes and determination of association and 
differential expression potential of gene haplotypes with the studied PE and SYP traits were performed in accordance with Kujur et al. (2015, 2015b) and M. S. Saxena, Bajaj, Das, et al. (2014).

\section{3 | RESULTS}

3.1 | Large-scale SNP genotyping discovers genomewide and photosynthesis pathway gene-derived novel alleles for genomics-assisted breeding applications of chickpea

The large-scale genotyping of SNPs discovered from 136 photosynthesis metabolic pathways-related genes among 92 desi and kabuli accessions representing an association panel detected 3,338 highquality SNPs from different coding and noncoding sequence components of these genes with a mean frequency of 24.5 SNPs/gene (Table S2). The sequencing of 96-plex ApeKI GBS libraries constructed from 92 accessions (with an average of 2.1 million reads per chickpea accession) produced 207.9 million high-quality sequence reads. Of these, 149.8 million sequence reads produced from chickpea accessions were mapped on kabuli reference genome according to their unique physical position (bp). The sequencing information obtained in this study was submitted to a NCBI-short read archive database (www.ncbi.nlm.nih.gov/sra) with accession number SRX845396 for unrestricted public access. In total, 11,079 high-quality SNPs (with $\geq 10$ read-depth and $\geq 20$ SNP base quality in individual accession) were detected from 92 accessions using kabuli reference (4,314 SNPs)- and de novo (6,765)-based GBS strategy (Figure 1a,b; Table S2). Notably, 3,651 and 663 reference-based SNPs were mapped on eight chromosomes and unanchored scaffolds of kabuli chickpea genome, respectively. This underlines greater utility of GBS assay in fast high-throughput discovery and genotyping of high-quality SNPs altogether at a whole genome level in chickpea. In this context, GBS-derived genome-wide SNPs discriminating domesticated desi and kabuli accessions discovered in the current study have much implications for their immense use and broader practical applications in genomics-assisted breeding and further genetic enhancement studies of chickpea.

Notably, 7,652 including 3,338 gene-derived and 4,314 genome-wide GBS-SNPs were mapped across chromosomes (6,826 SNPs) and unanchored scaffolds (826) of kabuli genome (Figure 1a, b; Table S2). The highest number of 907 SNPs (21\%) discovered from 291 genes were mapped on Chromosome 4, whereas maximum of 613 (18.4\%) genome-wide SNPs were localized on Chromosome 1 . The detailed structural annotation of 3,338 gene-based and 4,314 genome-wide SNPs demonstrated the occurrence of 5,933 (77.5\%) and 1,719 (22.5\%) SNPs in 1,410 genes and intergenic regions, respectively (Figure 1b,c; Table S2). The gene-derived SNPs included the highest and lowest proportion of $42.7 \%$ (2531) and $0.3 \%(20)$ SNPs in the introns and URRs of 631 and 4 genes, respectively. The 1,901 coding SNPs consisted of 59\% (1,130 SNPs) and $41 \%$ (771) synonymous and nonsynonymous (missense/nonsense) SNPs, respectively (Figure 1b,c; Table S2). The functional
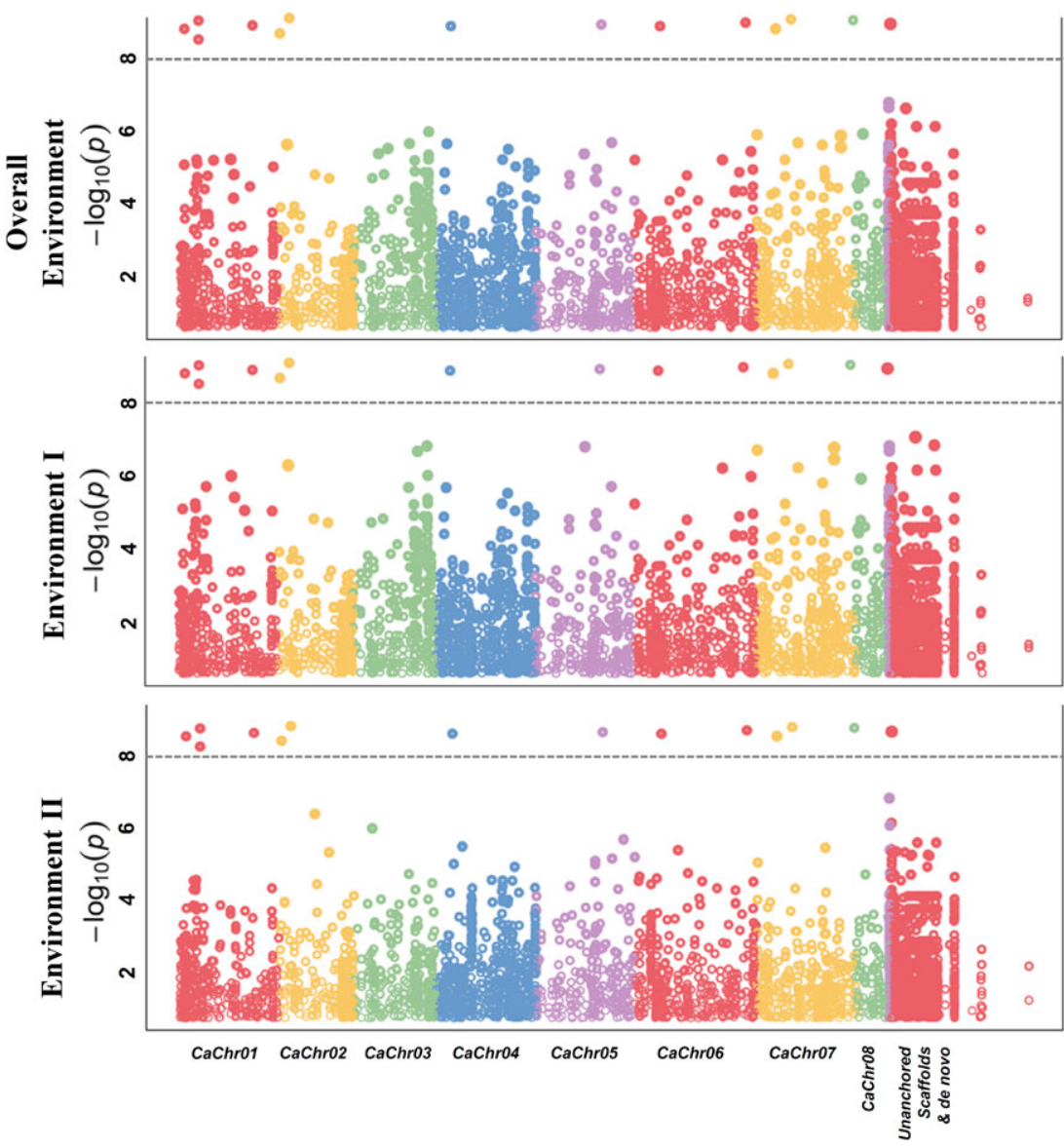

FIGURE 2 The use of 14,417 single nucleotide polymorphisms (SNPs) in genomewide association study produced a Manhattan plot depicting the significant $p$ value of 16 genomic SNP loci associated with five photosynthetic efficiency (chlorophyll content, SPAD chlorophyll meter reading, chlorophyll fluorescence, $\mathrm{CO}_{2}$ assimilation rate at increasing $\mathrm{CO}_{2}$ concentration, and $\mathrm{CO}_{2}$ assimilation rate at increasing light intensity) and seed yield traits in chickpea. The genomic distribution of reference genome-derived and de novo SNPs mapped on eight chromosomes and scaffolds of kabuli genome are indicated by the $x$ axis. The $y$ axis designates the $-\log _{10}$ (p) value for significantly associated SNP loci with photosynthetic efficiency and seed yield traits. The SNPs exhibiting significant association with the studied traits at a cut-off $p$ value $\leq 10^{-8}$ are demarcated with a dotted line [Colour figure can be viewed at wileyonlinelibrary.com] 
TABLE 3 Molecular mapping of major photosynthetic efficiency and seed yield QTLs in chickpea

$\begin{array}{llll} & & & \\ \text { QTLs }^{\text {a }} & \text { LGs/ Chromosomes } & \begin{array}{l}\text { Marker intervals with } \\ \text { genetic positions (cM) }\end{array} & \text { QTL physical intervals (bp) } \\ \text { CaqPE1.1 } & \text { CaLG(Chr)1 } & \text { CakSNP807(C/T): } 20.54 \text { to } & \text { CakSNP807(C/T): 7,050,958 to } \\ & & \text { CakSNP1313 (G/C): } 26.40 & \text { CakSNP1313 (G/C): 13,680,008 }\end{array}$

$\begin{array}{llr}\text { CaqPE1.2 } & \text { CaLG(Chr)1 } & \begin{array}{r}\text { CakSNP1875(T/G): } 78.45 \text { to } \\ \text { CakSNP2061 (C/T): } 84.70\end{array} \\ \text { CaqPE2.1 } & \text { CaLG(Chr)2 } & \begin{array}{r}\text { CakSNP2892(A/G): } 10.56 \text { to } \\ \text { CakSNP2899 (T/C): } 14.89\end{array} \\ \text { CaqPE2.2 CaqSYP2.1 } & \text { CaLG(Chr)2 } & \begin{array}{r}\text { CakSNP3281(T/C): } 57.80 \text { to } \\ \text { CakSNP3283 (C/A): } 61.53\end{array} \\ & & \text { CakSNP5697(C/T): } 22.45 \text { to } \\ \text { CaqPE4.1 } & \text { CaLG(Chr)4 } & \text { CakSNP5710 (A/C): } 26.48 \\ & & \text { CakSNP8486(G/A): } 3.14 \text { to } \\ \text { CaqPE5.1 } & \text { CakSNP8622 (T/C): } 5.68 \\ & \text { CaLG(Chr)5 } & \text { CakSNP13971(G/A): } 32.54 \text { to } \\ \text { CaqPE6.1 } & & \text { CakSNP10345(T/C): } 36.81 \\ & \text { CaLG(Chr)6 } & \text { CakSNP11526(A/T): } 62.12 \text { to } \\ & & \text { CakSNP11598(G/A): } 66.78 \\ \text { CaqPE6.2 } & & \text { CakSNP12106(T/C): } 53.45 \text { to } \\ & & \text { CakSNP12681(C/T): } 59.12\end{array}$

CakSNP1875(T/G): $40,358,747$ to
CakSNP2061 (C/T): 46,920,524
CakSNP2892(A/G): $30,335,194$ to
CakSNP2899 (T/C): $30,370,411$

CakSNP3281(T/C): 35,601,657 to CakSNP3283 (C/A): 35,612,587

CakSNP5697(C/T): 8,669,515 to

CakSNP8486(G/A): $31,176,844$ to CakSNP8622(T/C): 33,673,894

CakSNP13971(G/A): 11,665,233 to CakSNP10345(T/C): 15,861,278

CakSNP11526(A/T): 58,237,503 to
CakSNP11598(G/A): 58,908,024

CakSNP12106(T/C): 7,580,158 to CakSNP12681(C/T): 20,496,303 CakSNP5710 (A/C): 8,805,578
Markers tightly linked to QTLs with genetic (cM) and physical positions (bp)

Ca17446222(T/C): 21.86 and 7,446,222 Ca112641213(C/T): 23.87 and $12,641,213$ Ca113563280(G/C): 25.53 and $13,563,280$

\section{Ca142189380(A/G): 81.49 and $42,189,380$ \\ Ca2245818(A/G): 11.83 and 245,818}

Ca23343577(A/G): 58.27 and $3,343,577$

Ca48779743(C/G): 24.17 and $8,779,743$

Ca533245382(C/A): 3.97 and $33,245,382$

Ca614019110(G/C): 34.89 and $14,019,110$

\section{Ca658843981(A/T): 64.87 and $58,843,981$ \\ Ca78419380(A/G): 55.87 and 8,419,380 Ca710909055(A/G): 57.34 and $10,909,055$ Ca711324664(C/G): 58.17 and 11,324,664 Ca719530542(T/G): 58.95 and $19,530,542$}

Note. $\mathrm{LGs}=$ linkage groups; $\mathrm{cM}=$ centiMorgan; $\mathrm{PE}=$ photosynthetic efficiency; $\mathrm{SYP}=$ seed yield per plant; $\mathrm{LOD}=$ logarithm of odds; $\mathrm{PVE}=$ phenotypic variation explained; $\mathrm{DRR}=$ downstream regulatory region; $\mathrm{CC}=$ chlorophyll content; $\mathrm{SCMR}=\mathrm{SPAD}$ chlorophyll meter reading; $\mathrm{CF}=$ chlorophyll fluorescence; $\mathrm{CAR} \uparrow \mathrm{CO}_{2}=$ assimilation rate at increasing $\mathrm{CO}_{2}$ concentration; $\mathrm{CAR} \uparrow \mathrm{LI}=\mathrm{CO}_{2}$ assimilation rate at increasing light intensity; $\mathrm{A}=$ additive effect.

${ }^{a}$ CaqPE1.1 (Cicer arietinum QTL for photosynthetic efficiency on Chromosome 1 Number 1 ) and CaqSYP2.1 (C. arietinum QTL for seed yield per plant on Chromosome 2 Number 1). Proportion of PVE by QTLs; PE positive additive effect infers alleles from high PE and SYP mapping parental chickpea accession ICC 4958. Details regarding SNPs are mentioned in the Tables S2 and S5.

annotation of 1,410 genes with 7,652 SNPs exhibited their highest correspondence with the protein-coding genes related to KEGG photosynthesis pathway module of Calvin cycle (27\%, 909 SNPs in 34 genes) followed by Photosystem II (25\%, 846 SNPs in 37 genes; Figure 1d; Table S2).

\subsection{Association mapping identifies potential genomic loci governing PE and SYP traits in chickpea}

To perform gene-based association mapping and GWAS, we employed genome-wide GBS (11,079 SNPs)- and gene-based (3,338 SNPs) SNP genotyping data of 14,417 SNPs assayed in 92 chickpea accessions (association panel; Table S2). The use of these 14,417 SNPs, in determining high-resolution population structure and PCA, discriminated all 92 desi and kabuli accessions from each other and overall grouped into two distinct populations, POP I and POP II. The LD decay estimation in an association panel using 7,652 SNPs (4,314 genome-wide and 3,338 gene-based SNPs) that were mapped across eight chromosomes, illustrated LD decay $\left(r^{2}\right.$ reduced to half of its highest value) nearly at 200-300 kb physical distance of chromosomes. A wider level of phenotypic variation (2.91-24.81\% CV) and normal frequency distribution of $\mathrm{PE}$ (CC, SCMR, CF, CAR $\uparrow \mathrm{CO}_{2}$, and CAR $\uparrow$ LI) and SYP traits in an association panel representing two population groups based on multienvironment field phenotyping data was apparent (Table 1; Figure S1). All accessions representing an association panel revealed high $\mathrm{H}^{2}$ (varied from $80 \%$ to $85 \%$ ) for the six studied traits across environments. Maximum significant $(p<.0001)$ positive correlation among five PE traits (mean $r$ : 0.97) and minimum positive correlation between PE and SYP traits (0.61) were observed in an association panel. A higher significant difference $(p<.0001)$ among 92 
TABLE 3 (Continued)

\begin{tabular}{|c|c|c|c|c|c|c|}
\hline \multirow[b]{2}{*}{$Q_{T L}^{a}$} & \multirow{2}{*}{$\begin{array}{l}\text { Structural and functional } \\
\text { annotation of QTL-linked } \\
\text { markers }\end{array}$} & \multicolumn{4}{|c|}{ M-QTLs (Main effect QTLs) } & \multirow{2}{*}{$\begin{array}{l}\text { Epistatic } \\
\text { QTLs (E-QTLs) } \\
\text { PVE }\left(R^{2} \%\right)\end{array}$} \\
\hline & & $\begin{array}{l}\text { PE and SYP } \\
\text { traits-associated }\end{array}$ & LOD & PVE (R²\%) & A & \\
\hline CaqPE1.1 & $\begin{array}{l}\text { Ca_07996 (nonsynonymous) } \\
\text { Oxidoreductase FAD/NAD(P) } \\
\text { Ca_02491 (nonsynonymous) } \\
\text { Phosphoenolpyruvate } \\
\text { carboxylase Ca_14123 (DRR) } \\
\text { Iron-sulphur protein }\end{array}$ & $\begin{array}{l}\mathrm{CC}, \mathrm{SCMR}, \mathrm{CF} \\
\mathrm{CAR} \mathrm{CO}_{2}, \\
\text { and CAR个LI }\end{array}$ & 7.6 & 10.3 & 5.2 & 28.6 \\
\hline CaqPE1.2 & $\begin{array}{l}\text { Ca_22679 (DRR) Glyceraldehyde } \\
\text { 3-phosphate dehydrogenase }\end{array}$ & $\begin{array}{l}\mathrm{CC}, \mathrm{SCMR}, \mathrm{CF}, \\
{\mathrm{CAR} \uparrow \mathrm{CO}_{2},} \\
\text { and CAR个LI }\end{array}$ & 5.8 & 10.5 & 5.1 & 31.7 \\
\hline CaqPE2.1 & $\begin{array}{l}\text { Ca_16978 (DRR) Ribulose } \\
\text { bisphosphate carboxylase }\end{array}$ & 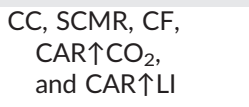 & 6.0 & 10.3 & 3.8 & 38.5 \\
\hline CaqPE2.2 CaqSYP2.1 & $\begin{array}{l}\text { Ca_10519 (nonsynonymous) } \\
\text { Chlorophyll A-B binding protein }\end{array}$ & $\begin{array}{l}\text { CC, SCMR, CF, } \\
\text { CAR } \mathrm{CO}_{2}, \\
\text { CAR } \uparrow \text { LI, and SYP }\end{array}$ & $\begin{array}{l}11.7 \text { (PE) } \\
11.2 \text { (SYP) }\end{array}$ & $\begin{array}{l}20.8 \text { (PE) } \\
20.1 \text { (SYP) }\end{array}$ & $\begin{array}{l}6.7 \text { (PE) } \\
6.3 \text { (SYP) }\end{array}$ & - \\
\hline CaqPE4.1 & $\begin{array}{l}\text { Ca_08373 (nonsynonymous) } \\
\text { Lactate/malate dehydrogenase }\end{array}$ & $\begin{array}{l}\mathrm{CC}, \mathrm{SCMR}, \mathrm{CF}, \\
\mathrm{CAR} \mathrm{CO}_{2}, \\
\text { and CAR个LI }\end{array}$ & 5.3 & 8.6 & 5.0 & 29.7 \\
\hline CaqPE5.1 & $\begin{array}{l}\text { Ca_05017 (nonsynonymous) } \\
\text { Malic oxidoreductase }\end{array}$ & $\begin{array}{l}\mathrm{CC}, \mathrm{SCMR}, \mathrm{CF} \\
\mathrm{CAR} \mathrm{CO}_{2} \\
\text { and CAR } \mathrm{CAI}\end{array}$ & 5.0 & 9.5 & 4.8 & 26.3 \\
\hline CaqPE6.1 & $\begin{array}{l}\text { Ca_05167 (DRR) Chlorophyll } \\
\text { A-B binding protein }\end{array}$ & $\begin{array}{l}\mathrm{CC}, \mathrm{SCMR}, \mathrm{CF}, \\
\mathrm{CAR} \uparrow \mathrm{CO}_{2}, \text { and } \\
\text { CAR } \uparrow \mathrm{LI}\end{array}$ & 6.5 & 8.8 & 4.1 & - \\
\hline CaqPE6.2 & $\begin{array}{l}\text { Ca_15426 (nonsynonymous) } \\
\text { Fructose-bisphosphate aldolase }\end{array}$ & $\begin{array}{l}\text { CC, SCMR, CF, } \\
\text { CAR } \uparrow \mathrm{CO}_{2} \text {, and } \\
\text { CAR } \uparrow \mathrm{LI}\end{array}$ & 6.2 & 10.2 & 3.9 & 27.8 \\
\hline CaqPE7.1 CaqSYP7.1 & $\begin{array}{l}\text { Ca_13165 (nonsynonymous) } \\
\text { Timing of CAB Expression } \\
1 \text { Ca_12847 (nonsynonymous) } \\
\text { Ferredoxin Ca_09362 (nonsynonymous) } \\
\text { Basic-leucine zipper (bZIP) Ca_12348 } \\
\text { (nonsynonymous) } \\
\text { Phosphoenolpyruvate carboxykinase }\end{array}$ & $\begin{array}{l}\text { CC, SCMR, CF, } \\
\text { CAR } \uparrow \mathrm{CO}_{2}, \\
\text { CAR } \uparrow \mathrm{LI}, \text { and } \\
\text { SYP }\end{array}$ & $\begin{array}{l}10.1 \text { (PE) } \\
10.5 \text { (SYP) }\end{array}$ & $\begin{array}{l}13.0 \text { (PE) } \\
19.7 \text { (SYP) }\end{array}$ & $\begin{array}{l}5.4 \text { (PE) } \\
\quad 4.8 \text { (SYP) }\end{array}$ & 43.541 .6 \\
\hline
\end{tabular}

accessions (association panel) both for PE (CC, SCMR, CF, CAR个CO 2 , and CAR个LI) and SYP traits was observed despite substantial environmental effect on these traits (Table S3). A significant interaction between genotypes (G)/accessions and environment ( $E$ ) for the said traits based on $G \times E$ variance was apparent.

For gene-based association mapping and GWAS, genotyping data of 14,417 SNPs assayed among 92 accessions (association panel) were integrated with their multienvironments field phenotyping data of PE (CC, SCMR, CF, CAR $\mathrm{CO}_{2}$, and CAR个LI) and SYP traits. The CMLM-led association study at a significant FDR cut-off $\leq 0.05$ detected 16 genomic loci revealing association with six $\mathrm{PE}$ (CC, SCMR, CF, CAR $\uparrow \mathrm{CO}_{2}$, and CAR个LI) and SYP traits at a $p \leq 10^{-8}$ which were got validated across two environments (Table 2; Figure 2). The association potential of genomic SNP loci for PE and SYP traits estimated for entire population remained intact (based on their identities and physical locations) when analysed in two chickpea populations (POP I and POP II) individually. Fourteen trait-associated SNP loci were localized on seven chromosomes (beside Chromosome 3), whereas remaining two SNP loci were mapped on two unanchored scaffolds of chickpea genome (Table 2; Figure 2). The highest four trait-associated SNPs were mapped on Chromosome 1 followed by three SNPs on Chromosome 7. Ten and 6 of 16 trait-associated loci were derived from diverse coding (10 nonsynonymous SNPs) and noncoding (one URR-SNP and five DRR-SNPs) regions of 16 genes, respectively (Table 2; Figure 2). Thirteen SNPs derived from different coding and noncoding sequence components of 13 genes were associated with five $\mathrm{PE}$ (CC, SCMR, CF, CAR $\uparrow \mathrm{CO}_{2}$, and CAR $\uparrow$ LI) traits, whereas three nonsynonymous coding SNPs from three genes were associated with both PE and SYP traits. The PVE determined by 16 PE and SYP traitassociated individual loci of 16 genes among 92 chickpea accessions varied from $5.0 \%$ to $12.0 \% R^{2}$. All these 16 trait-associated loci altogether in combination gave $18.4 \%$ PVE. Three individual nonsynonymous SNPs-containing three genes showing significant association with both PE and SYP traits revealed 10.2-12.0\% PVE (combined PVE: 20.6\%), whereas 13 PE-associated individual genebased SNPs revealed 5.0-6.3\% PVE (10.2\%) in chickpea. We observed a strong association of one coding SNP (A/G) revealing nonsynonymous amino acid substitution (Isoleucine [ATC] to Valine [GTC]) in a chlorophyll A-B binding protein-coding gene $\left(1.2 \times \leq 10^{-9} \mathrm{P}\right.$ with $\left.12 \% R^{2}\right)$ as compared with other 15 identified genomic loci with PE (CC, SCMR, CF, CAR $\mathrm{CO}_{2}$, and CAR $\uparrow \mathrm{LI}$ ) and SYP traits. Henceforth, this gene was considered as a promising candidate for dissection of PE and SYP traits in chickpea. 


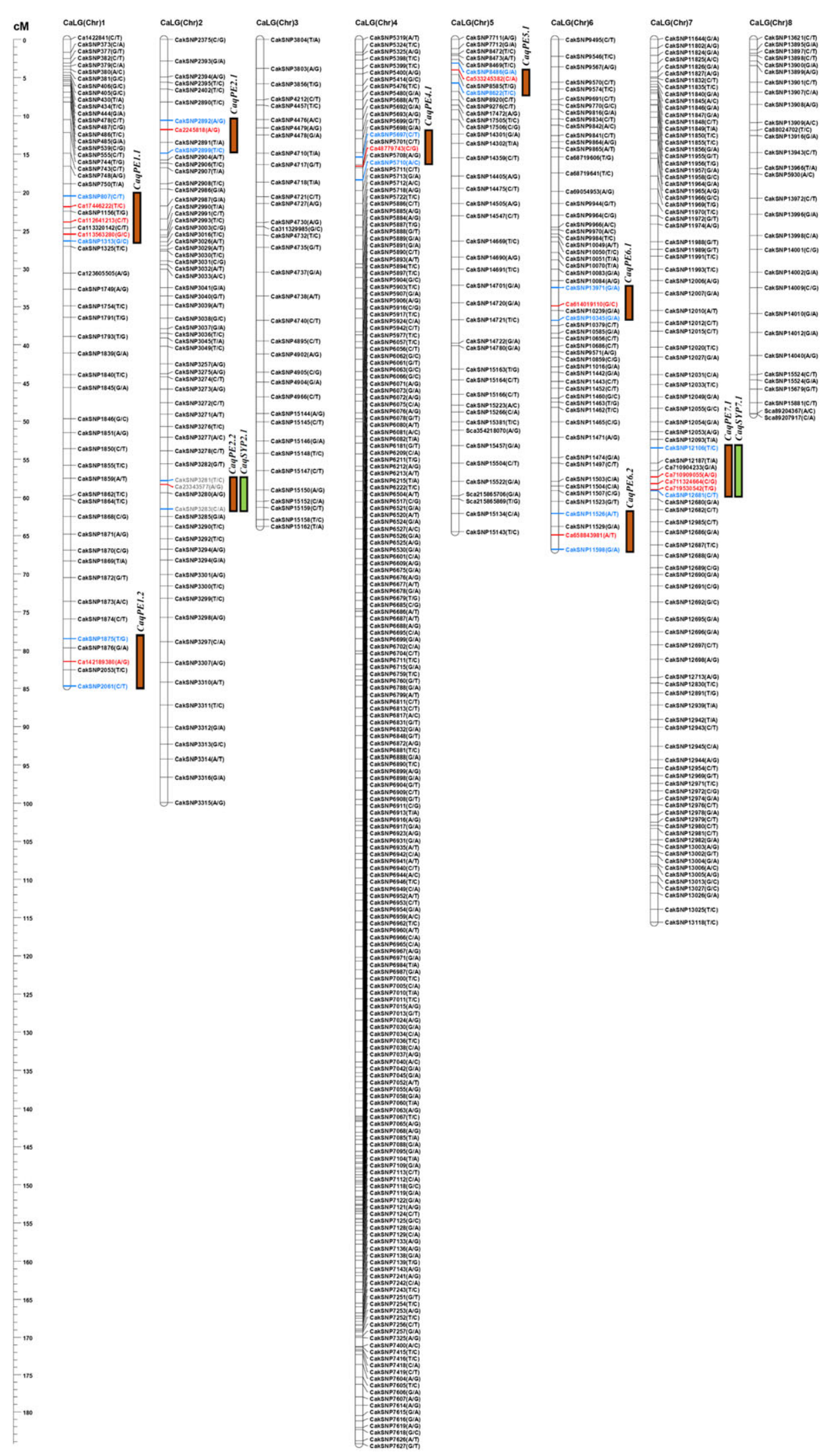

FIGURE 3 Nine genomic regions harbouring 11 major QTLs associated with five photosynthetic efficiency (chlorophyll content, SPAD chlorophyll meter reading, chlorophyll fluorescence, $\mathrm{CO}_{2}$ assimilation rate at increasing $\mathrm{CO}_{2}$ concentration, and $\mathrm{CO}_{2}$ assimilation rate at increasing light intensity) and seed yield traits mapped on six chromosomes of an intraspecific high-density genetic linkage map (ICC $4958 \times$ ICC 12299) of chickpea. The genetic distance (cM [centiMorgan]) and identity of the single nucleotide polymorphism (SNP) loci mapped on chromosomes are denoted on left and right sideways of chromosomes, respectively. The details of SNPs flanking and tightly linked to major QTLs are provided in the Tables 3 and S5. Orange and yellow square boxes represent the QTLs associated with photosynthetic efficiency and seed yield traits, respectively, mapped on chromosomes of a genetic map. The SNPs flanking and tightly linked to major QTLs are depicted with blue and red colour lines, respectively 


\section{3 | Molecular mapping of QTLs ascertains} association potential of genomic loci for PE and SYP traits in chickpea

We constructed an intraspecific genetic map (ICC $4958 \times$ ICC 12299) of chickpea by integrating 589 SNPs across eight LGs (LG1-LG8; Tables S4 and S5). The genetic linkage map covered a total map length of $728.65 \mathrm{cM}$ with a map density that defined as mean intermarker distance of $1.24 \mathrm{cM}$ (Table 3; Figure 3). The highest and lowest saturated genetic maps were LG4 and LG3 with the map densities of 0.90 and 1.82 , respectively. This high-density map characteristic of a constructed genetic linkage map reflected its utilization in high-resolution QTL mapping of agronomic traits in chickpea. The field phenotyping of a RIL mapping population (ICC $4958 \times$ ICC 12299) exhibited a wider level of PE (CC, SCMR, CF, CAR $\uparrow \mathrm{CO}_{2}$, and CAR $\uparrow$ LI) and SYP trait variation (3.06-28.57\% CV) and high $\mathrm{H}^{2}$ (80-83\%) among 236 mapping individuals and parental accessions across two environments (Table 1; Figure S1). The normal frequency distribution including bidirectional transgressive segregation of these studied traits in RILs emphasizes the higher proficiency of a generated RIL population (ICC 4958 × ICC 12299) for subsequent QTL mapping study in chickpea (Figure S1). We observed a significant difference $(p<.0001)$ among RIL mapping individuals for PE (CC, SCMR, CF, CAR $\uparrow \mathrm{CO}_{2}$, and CAR $\uparrow L I$ ) and SYP traits even though these traits were influenced by significant environmental effect as evident from $G \times E$ interaction variance (Table S3).

The QTL mapping was performed by combining genotyping information of 589 genetically mapped SNPs and two environments PE and SYP trait field phenotyping data of RIL mapping individuals along with parental accessions. This detected $11 \mathrm{M}$-QTLs (5.0-11.7 LOD) harbouring nine genomic regions governing PE and SYP traits were mapped on six chickpea LGs/chromosomes (except 3 and 8; Table 3; Figure 3). The PVE determined by individual M-QTL for PE and SYP traits varied from $8.8-20.8 \% R^{2}$. The PVE measured for all $11 \mathrm{M}$-QTLs in combination was $24.8 \%$. All these $11 \mathrm{M}$-QTLs were got validated across two environments evaluated and thereby considered as robust QTLs governing PE and SYP traits in chickpea. Seven M-QTLs associated with PE traits (8.6-10.5 PVE with 5.07.6 LOD) were mapped on seven different unique genomic regions of five chromosomes (Table 3; Figure 3 ). The remaining four $\mathrm{M}$ QTLs governing both PE (13.0 to 20.8 PVE with 10.1 to 11.7 LOD) and SYP (19.7 to 20.1 PVE with 10.5 to 11.2 LOD) traits were mapped on the identical genomic regions of Chromosomes 2 and 7. The detected $11 \mathrm{M}-\mathrm{QTLs}$ exhibited positive additive gene effect for PE (3.8-6.7) and SYP (4.8-6.3) traits inferring the effective contribution of alleles derived from a high PE and SYP mapping parental accession ICC 4958 on these loci for enhancing the target traits (Table 3). Three-loci interaction Genotype Matrix Mapping-based QTL analysis detected $28 \mathrm{E}-\mathrm{QTL}$ for PE and SYP traits of which nine E-QTLs (26.3-43.5\% PVE) corresponded to M-QTLs mapped on chromosomes (Table 3). The effective integration of our association and QTL mapping outcomes revealed that SNPs annotated from 14 candidate genes linked to 11 major M-QTLs had potential for significant association with PE and SYP traits in chickpea (Tables 2 and 3). Notably, one coding SNP (A/G) revealing nonsynonymous amino acid substitution (Isoleucine [ATC] to Valine [GTC]) in a chlorophyll A-B binding protein-coding gene mapped on a 3,343,577 BP (58.27 cM) genomic interval of CaqPE2.2 and CaqSYP2.1 M-QTLs exhibited strong association potential for both PE and SYP traits based on association analysis (12.0\% PVE with $1.2 \times 10^{-9} \mathrm{P}$ ) and QTL mapping (20.8\% PVE with 11.7 LOD; Tables 2 and 3). Moreover, at these two CaqPE2.2 and CaqSYP2.1 M-QTL regions, maximum positive additive effects and thus highest positive interactions of QTLs/alleles governing both enhanced PE and SYP traits derived from a high PE and SYP mapping parental accession ICC 4958 was evident. In these perspectives, nonsynonymous SNP allelic variants of a chlorophyll A-B binding protein-coding gene governing both PE (CC, SCMR, CF, $\mathrm{CAR} \uparrow \mathrm{CO}_{2}$, and $\mathrm{CAR} \uparrow \mathrm{LI}$ ) and SYP traits tightly linked to CaqPE2.2 and CaqSYP2.1 major QTLs (validated by GWAS and QTL mapping), respectively, was selected as potential candidate for further validation by transcript profiling in chickpea.

\section{4 | Differential expression profiling validates regulatory function of associated genes for PE and SYP traits in chickpea}

The differential expression profiling of $16 \mathrm{PE}$ and SYP trait-associated genes (validated through association and QTL mapping) was performed in multiple vegetative and reproductive tissues (shoot, root, young/mature leaf, flower bud, young pod, and seed) of parental accessions (ICC 4958 and ICC 12299) and two of each homozygous individuals from a RIL mapping population (ICC $4958 \times$ ICC 12299) using quantitative RT-PCR assay (Table 2). Eight desi and kabuli germplasm accessions with contrasting PE and SYP traits were also included for expression profiling (Table S6). All $16 \mathrm{PE}$ and SYP trait-associated genes were significantly up-regulated ( $\geq$ fourfold) in young/mature leaves (third/fourth leaf of 30-40 DAS grown plants) as compared with respective vegetative and reproductive tissues of germplasm accessions, RIL individuals, and mapping parents (Table 2; Figure 4). The up-regulation ( $\geq$ threefold) of all 16 trait-associated genes in high than that of low PE and SYP RILs and accessions was observed. Four genes with nonsynonymous and regulatory SNPs were extremely up-regulated $(\geq 10$-fold) in leaves of high than that of low PE and SYP RILs and accessions (Table 2; Figure 4). Notably, a chlorophyll A-B binding protein-coding gene with a nonsynonymous SNP revealing strong association with PE and SYP traits exhibited pronounced up-regulation (14.9-fold) in young/mature leaves of high as compared with low PE and SYP RIL mapping individuals, parents, and germplasm accessions (Table 2; Figure 4).

\subsection{Molecular haplotyping identifies natural allelic variants and superior haplotypes of interacting genes governing PE and SYP traits in chickpea}

The in silico protein-protein interaction study enabled to identify eight Arabidopsis homologues of chickpea genes possibly interacting with a strong PE and SYP trait-associated chlorophyll A-B binding protein-coding gene. The high-resolution candidate gene-based 


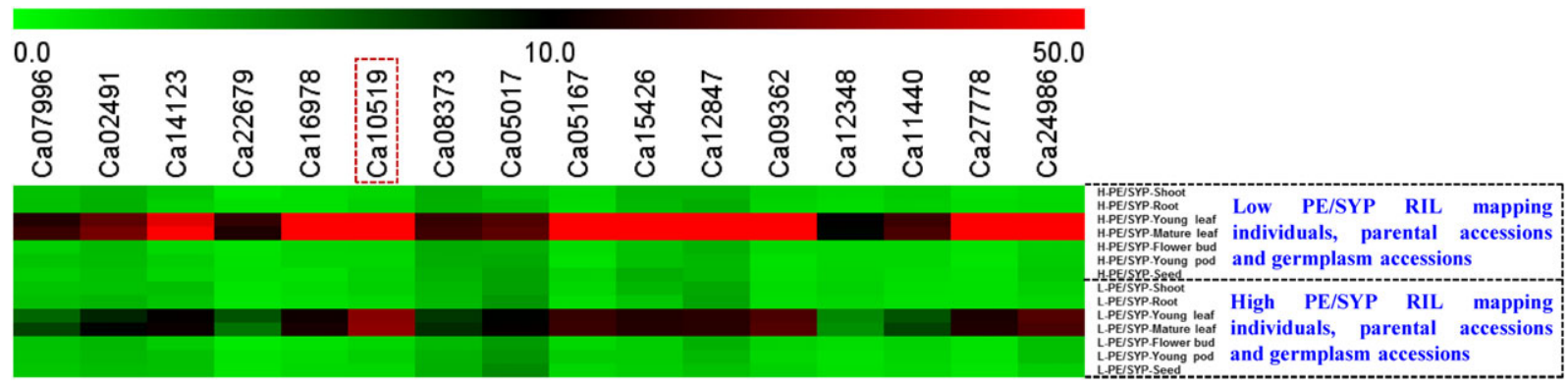

FIGURE 4 Differential expression profiles of 16 PE and SYP trait-associated genes (validated by high-resolution association analysis and QTL mapping) in vegetative/reproductive tissues (root, shoot, young leaf, mature leaf, flower bud, young pod, and seed) of germplasm accessions and homozygous mapping individuals and parental accessions of a recombinant inbred line (RIL) mapping population (ICC $4958 \times$ ICC 12299 ) contrasting with high and low PE and SYP traits using quantitative real time polymerase chain reaction assay. The green, black, and red color in colour scale at the top represent low, medium, and high level of average log signal expression value of genes in different tissues, respectively. A strong PE and SYP trait-associated gene exhibiting enhanced up-regulation especially in young and mature leaf of accessions and RIL mapping individuals contrasting with high photosynthetic efficiency and seed yield is highlighted with red box. The detail information regarding genes used for expression study are mentioned in the Table 2. The tissues and genes selected for expression profiling are indicated on right and upper portion of expression map, respectively. H-PE/SYP and L-PE/SYP = high and low photosynthetic efficiency and seed yield per plant [Colour figure can be viewed at wileyonlinelibrary.com]

association analysis and QTL mapping were performed by integrating the genotyping information of 183 coding and noncoding SNPs discovered from these said eight genes with multienvironments $\mathrm{PE}$ and SYP trait field phenotyping data of 92 germplasm accessions (association panel) and 236 RIL mapping individuals of chickpea (Tables S1 and S7). This exertion detected one coding SNP (A/G) revealing nonsynonymous amino acid substitution (Isoleucine [ATT] to Valine [GTT]) in a TIMING OF CAB EXPRESSION 1 (TOC1) gene mapped on a 8,419,380 BP (55.87 cM) genomic interval underlying the CaqPE7.1 and CaqSYP7.1 major QTLs of an intraspecific high-density chickpea genetic linkage map (ICC 4958 × ICC 12299). These potential molecular tags also demonstrated strong association with both PE and SYP traits based on association analysis (11.0\% PVE with $\left.1.0 \times 10^{-9} \mathrm{P}\right)$ and QTL mapping (19.7 PVE with 10.5 LOD) performed in the current study.

The molecular haplotyping of a strong PE and SYP trait-associated chlorophyll A-B binding protein-coding gene (Ca_10519) and its interacting trait-associated gene TOC1 (Ca_13165) using 33 and 22 SNPs including two functionally relevant nonsynonymous trait-linked SNPs respectively constituted two major haplotypes for each gene with the high LD resolution (Figure $5 a-d$ ). The gene haplotype-specific association analysis identified two major haplotypes, HAP A (30\% to $37 \%$ PVE with $10^{-9}$ to $10^{-11} \mathrm{P}$ ) and HAP B (41\% to 49\% PVE with $10^{-12}$ to $10^{-14} \mathrm{P}$ ), from the CDS regions of each two genes represented by significant proportion of desi and kabuli germplasm accessions exhibiting strong association with low and high PE efficiency as well as SYP trait differentiation, respectively, in a constituted association panel (Figure 5e). The haplotype-specific differential expression profiling targeting these two potential trait-associated genes (chlorophyll A-B binding protein-coding gene and TOC1) depicted pronounced up-regulation (>sevenfold) of HAP B in the young/mature leaves of germplasm accessions contrasting with high PE and SYP traits as compared with that of HAP A in the accessions contrasting with low PE and SYP traits (Figure 5f). This implicates the functional significance of natural allelic variants and haplotypes identified from two genes in regulating PE traits contributing for seed yield enhancement of chickpea.

\section{4 | DISCUSSION}

\subsection{Integrated genomic strategy delineates functionally relevant molecular signatures regulating PE and SYP traits in chickpea}

The present study integrated gene-based association mapping and GWAS with high-resolution QTL mapping, expression profiling, and molecular haplotyping to narrow down the genomic loci (genes and alleles) influencing PE and SYP traits in chickpea. Chickpea has undergone four successive evolutionary bottlenecks during its course of domestication resulting in narrow genetic base, low intraspecific polymorphism, and extended LD decay in cultivated genepool (Abbo, Gopher, Rubin, \& Lev-Yadun, 2005; Berger, Buck, Henzell, \& Turner, 2005; Penmetsa et al., 2016; Toker, 2009). Thus, integrating GWAS with gene-based association analysis will facilitate the dissection of complex agronomic traits in chickpea. Our study utilized this strategy to scan the most promising genomic loci governing PE and SYP traits in chickpea. For this, the natural SNP allelic variants (14,417 SNPs) scanned from whole genome including 136 photosynthesis metabolic pathway-related chickpea gene homologues of crop plants (Ambavaram et al., 2014; De Souza et al., 2017; Dhanapal et al., 2016; Yamori et al., 2016; Zhu et al., 2004) were correlated with multienvironment PE and SYP trait field phenotyping data of 92 desi and kabuli accessions belonging to an association panel. Sixteen genomic SNP loci derived from nonsynonymous coding and regulatory sequence components of 16 genes were found to be significantly associated with six major PE (CC, SCMR, CF, CAR $\uparrow \mathrm{CO}_{2}$, and CAR $\uparrow$ LI) and SYP traits across two environments in chickpea. These informative novel SNP allelic variants scanned from the genes associated with PE and SYP traits have greater functional relevance in chickpea. This could thus be deployed in establishing fast marker-trait association and scanning of promising molecular signatures (genes/ QTLs and alleles) influencing the studied agronomic traits in chickpea. The natural SNP allelic variants discovered from all of the identified genes are known to be involved in various metabolism modules, 

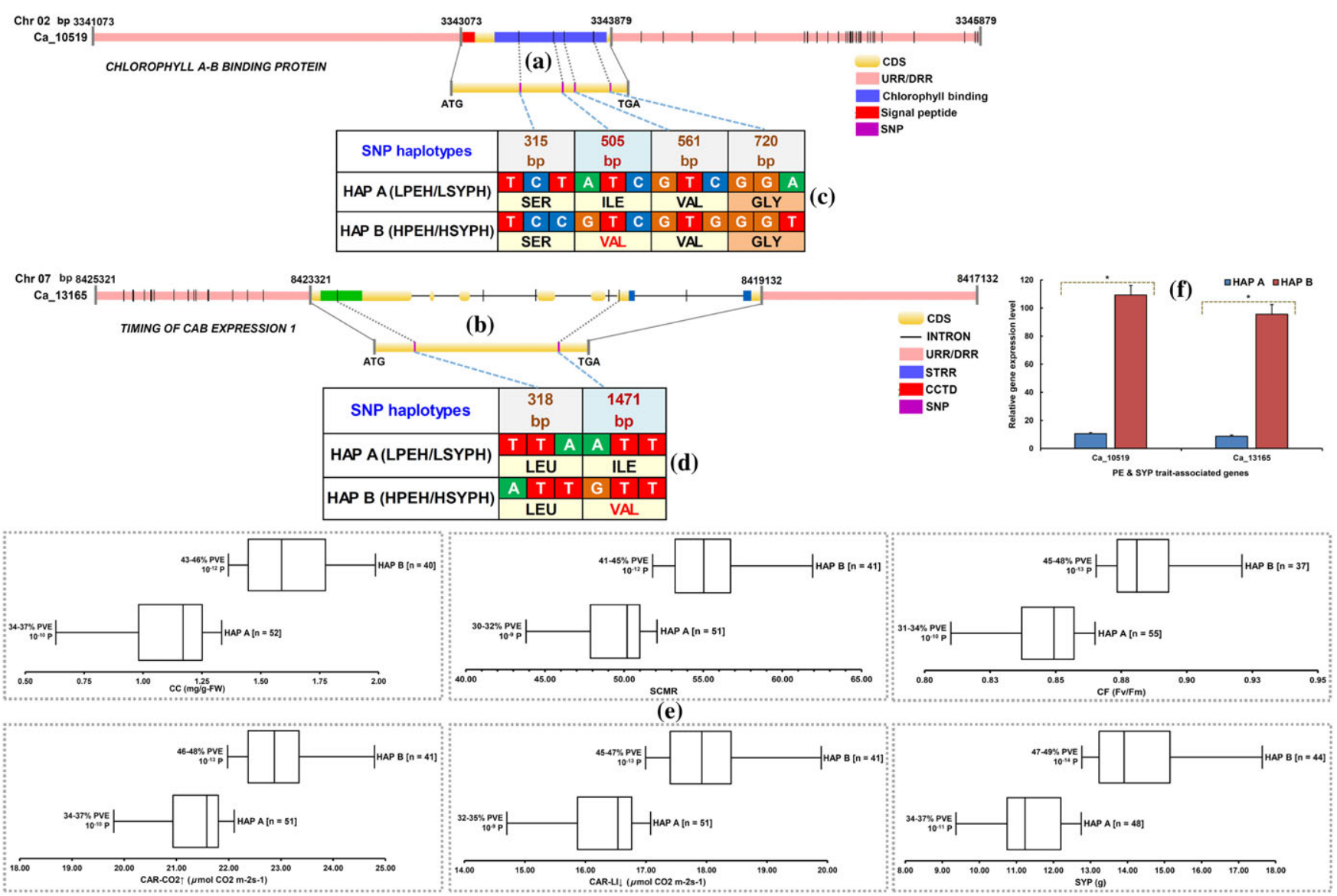

FIGURE 5 Constitution of haplotypes and their association mapping and expression profiling of a strong photosynthetic efficiency (PE) and seed yield per plant (SYP)-associated chlorophyll A-B binding protein-coding gene and its interacting gene, Timing of CAB Expression 1 (delineated by association analysis, QTL mapping, and expression profiling), validating potential of the gene haplotypes in regulating PE and SYP traits in chickpea. Genomic organization and constitution of a (a) chlorophyll A-B binding protein-coding gene and its interacting gene, (b) Timing of CAB Expression 1 exhibiting distribution of SNPs in different sequence components of these genes. (c,d) The genotyping of SNPs in different coding and noncoding sequence components of these two genes among 92 desi and kabuli cultivated chickpea accessions constituted two major haplotypes from each gene. (e) Two haplotypes, HAP A and HAP B, represented by the desi and kabuli accessions ( $n$ ) demonstrating strong association with low and high PE and SYP trait differentiation, respectively, are illustrated by the Box-Whisker Plots. (f) Haplotype-specific transcript profiling of two haplotypes constituted from chlorophyll A-B binding protein-coding gene (Ca_10519) and Timing of CAB Expression 1 (Ca_13165) gene using the young/mature leaf tissues of the two selected chickpea accessions representing low (HAP A) and high (HAP B) PE and SYP haplotypes. Error bars represent standard error $(n=3) .\left({ }^{*} p<.0001\right.$, two-tailed $t$ test). URR = upstream regulatory region; DRR = downstream regulatory region; $\mathrm{SNP}=$ single nucleotide polymorphism; $\mathrm{CC}=$ chlorophyll content; $\mathrm{SCMR}=\mathrm{SPAD}$ chlorophyll meter reading; $\mathrm{CAR} \downarrow \mathrm{LI}=\mathrm{CO}_{2}$ assimilation rate at decreasing light intensity; $\mathrm{CAR} \uparrow \mathrm{CO}_{2}=$ assimilation rate at increasing $\mathrm{CO}_{2}$ concentration; $\mathrm{STRR}=$ signal transduction response regulator; $\mathrm{CCTD}=$ CCT (CONSTANS, CONSTANS-like and TOC1) domain [Colour figure can be viewed at wileyonlinelibrary.com]

namely, F-type ATPase, crassulacean acid metabolism-light/dark, Cytochrome $b_{6} / f$-complex, calvin cycle, Photosystem II, and photosynthetic transporter of photosynthesis pathways in crop plants including chickpea. Among these, basic-leucine zipper (bZIP) transcription factor, protein kinase, and chlorophyll A-B binding protein exhibiting strong association ( $>10 \%$ PVE) with PE and SYP traits across two environments appear to be highly promising. The bZIP is known to govern transcriptional gene regulatory networks underlying growth, development, and abiotic stress responses in crop plants. The involvement of two bZIP transcription factors in transcriptional regulation of Rubisco activase gene (GmRCA $\alpha$ ) required for the light activation of most vital photosynthetic pathway enzyme, Ribulose-1,5bisphosphate carboxylase/oxygenase (Rubisco), is well documented in soybean (J. Zhang et al., 2016). The use of combinatorial genomic approach delineated a highly up-regulated chlorophyll A-B binding protein-coding gene influencing both PE and SYP traits in chickpea.
The functional significance of this gene with nonsynonymous SNP alleles in PE and SYP trait regulation was evident from its strong trait association potential and tight linkage with robust M-QTLs (CaqPE2.2 and CaqSYP2.1) governing the both enhanced PE and SYP traits positively. This is further supported well with young/mature leaf-specific expression of this gene especially in germplasm accessions and homozygous RIL mapping individuals with contrasting high PE and SYP traits. The chlorophyll A-B binding protein, belonging to the light harvesting complex of thylakoid membrane, functions in transfer of light energy to the reaction centre. The light harvesting chlorophyll binding (LHCB) proteins are reported to be involved in various growth, development, and drought stress responses in crop plants (Xu et al., 2012). An allelic variant of barley LHCB protein coding gene, Lhcb1, is known to be associated with a number of agro-economical traits (Xia et al., 2012). Five SNPs in Lhcb1 gene are found to be significantly associated with diverse agronomic traits including, 
spike length, grain number per spike, and thousand grain weight in barley (Xia et al., 2012).

A diverse array of proteins belonging mostly to photosystem complex has been reported to be interacting with the Arabidopsis homologue of our strong PE and SYP trait-associated chlorophyll A-B binding (CAB1) protein-coding gene. Among the chickpea homologues of these proteins, TOC1 was found to be strongly associated with photosynthesis efficiency and seed yield traits based on association analysis, QTL mapping, and expression profiling in chickpea. This gene is well characterized as a key regulator of circadian rhythm in Arabidopsis. Though direct interaction between TOC1 and CAB1 has not been reported but it regulates expression of $C A B 1$ through transcriptionally regulating LATE ELONGATED HYPOCOTYL and CIRCADIAN CLOCK ASSOCIATED 1. Both these genes are reported to be the positive regulator of $C A B 1$ and at the same time represses TOC1 expression during the day hours (Alabadí et al., 2001). These results hint a salient role of circadian rhythm for increasing PE in crop plants. The high-resolution association analysis, QTL mapping, and gene expression profiling combined with molecular haplotyping/LD mapping and haplotype-specific gene expression study delineated two superior haplotypes from each chlorophyll A-B binding protein-coding gene and its interacting TOC1 gene regulating both PE and SYP traits in chickpea. Higher heritability of these trait-associated molecular tags underlying robust QTLs across environments suggests their broader practical applicability in genetic enhancement studies of chickpea. This infers the functional relevance of molecular signatures scanned from these two potential genes for rapid dissection of complex PE and SYP traits in chickpea. A much comprehensive analysis of transcriptional regulation of aforesaid high PE and SYP trait-associated genes including two highly-promising genes and deciphering their subsequent role in control of photosynthesis metabolism pathway toward enhancing seed yield is essential for further deployment of the molecular tags in genomics-assisted crop improvement of chickpea. Though the current study utilized a small size association panel (92 accessions) for association mapping study, however, association potential of genes/alleles for PE and SYP traits was ascertained through high-resolution QTL mapping, expression profiling, and molecular haplotyping of interacting genes. Henceforth, the promising molecular signatures regulating PE and SYP traits delineated in the current study deploying an integrated genomics-assisted breeding strategy will be useful to develop high seed-yielding cultivars enriched with PE traits in chickpea.

\section{ACKNOWLEDGEMENTS}

The financial support provided by the Department of Biotechnology (DBT), Ministry of Science and Technology, Government of India, is acknowledged.

\section{ORCID}

Swarup K. Parida (D) http://orcid.org/0000-0001-7843-3031

\section{REFERENCES}

Alabadí, D., Oyama, T., Yanovsky, M. J., Harmon, F. G., Más, P., \& Kay, S. A. (2001). Reciprocal regulation between TOC1 and LHY/CCA1 within the Arabidopsis circadian clock. Science, 293, 880-883.
Abbo, S., Gopher, A., Rubin, B., \& Lev-Yadun, S. (2005). On the origin of near eastern founder crops and the 'dump-heap hypothesis'. Genetic Resources and Crop Evolution, 52, 491-495.

Ambavaram, M. M., Basu, S., Krishnan, A., Ramegowda, V., Batlang, U., Rahman, L., ... Pereira, A. (2014). Coordinated regulation of photosynthesis in rice increases yield and tolerance to environmental stress. Nature Communications, 5, 5302.

Bajaj, D., Saxena, M. S., Kujur, A., Das, S., Badoni, S., Tripathi, S., ... Parida, S. K. (2015). Genome-wide conserved non-coding microsatellite (CNMS) marker-based integrative genetical genomics for quantitative dissection of seed weight in chickpea. Journal of Experimental Botany, 66, 1271-1290.

Bajaj, D., Upadhyaya, H. D., Khan, Y., Das, S., Badoni, S., Shree, T., ... Parida, S. K. (2015). A combinatorial approach of comprehensive QTL-based comparative genome mapping and transcript profiling identified a seed weight-regulating candidate gene in chickpea. Scientific Reports, 5, 9264.

Benjamini, Y., \& Hochberg, Y. (1995). Controlling the false discovery rate: A practical and powerful approach to multiple testing. Journal of the Royal Statistical Society: Series B, 57, 289-300.

Berger, J. D., Buck, R., Henzell, J. M., \& Turner, N. C. (2005). Evolution in the genus Cicer vernalisation response and low temperature pod set in chickpea (C. arietinum L.) and its annual wild relatives. Australian Journal of Agricultural Research, 56, 1191-1200.

De Souza, A. P., Massenburg, L. N., Jaiswal, D., Cheng, S., Shekar, R., \& Long, S. P. (2017). Rooting for cassava: Insights into photosynthesis and associated physiology as a route to improve yield potential. New Phytologist, 213, 50-65.

Dhanapal, A. P., Ray, J. D., Singh, S. K., Hoyos-Villegas, V., Smith, J. R., Purcell, L. C., \& Fritschi, F. B. (2016). Genome-wide association mapping of soybean chlorophyll traits based on canopy spectral reflectance and leaf extracts. BMC Plant Biology, 16, 174.

Gautami, B., Pandey, M. K., Vadez, V., Nigam, S. N., Ratnakumar, P., Krishnamurthy, L., ... Varshney, R. K. (2012). Quantitative trait locus analysis and construction of consensus genetic map for drought tolerance traits based on three recombinant inbred line populations in cultivated groundnut (Arachis hypogaea L.). Molecular Breeding, 30, 757-772.

Gupta, S., Nawaz, K., Parween, S., Roy, R., Sahu, K., Pole, A. K., ... Chattopadhyay, D. (2016). Draft genome sequence of Cicer reticulatum L., the wild progenitor of chickpea provides a resource for agronomic trait improvement. DNA Research, 24, 1-10.

Jain, M., Misra, G., Patel, R. K., Priya, P., Jhanwar, S., Khan, A. W., ... Chattopadhyay, D. (2013). A draft genome sequence of the pulse crop chickpea (Cicer arietinum L.). Plant Journal, 74, 715-729.

Kang, H. M., Sul, J. H., Service, S. K., Zaitlen, N. A., Kong, S. Y., Freimer, N. B., ... Eskin, E. (2010). Variance component model to account for sample structure in genome-wide association studies. Nature Genetics, 42, 348-354.

Kashiwagi, J., Krishna, M. L., Singh, S., \& Upadhyaya, H. D. (2006). Variation of SPAD chlorophyll meter readings (SCMR) in the mini core germplasm collection of chickpea. Journal of Semi-Arid Tropical Agricultural Research, 2, 1-3.

Kale, S. M., Jaganathan, D., Ruperao, P., Chen, C., Punna, R., Kudapa, H., ... Varshney, R. K. (2015). Prioritization of candidate genes in "QTLhotspot" region for drought tolerance in chickpea (Cicer arietinum L.). Scientific Reports, 5, 15296.

Kujur, A., Bajaj, D., Upadhyaya, H. D., Das, S., Ranjan, R., Shree, T., ... Parida, S. K. (2015a). Employing genome-wide SNP discovery and genotyping strategy to extrapolate the natural allelic diversity and domestication patterns in chickpea. Frontiers in Plant Science, 6, 162.

Kujur, A., Bajaj, D., Upadhyaya, H. D., Das, S., Ranjan, R., Shree, T., ... Parida, S. K. (2015b). A genome-wide SNP scan accelerates traitregulatory genomic loci identification in chickpea. Scientific Reports, 5, 11166. 
Kujur, A., Upadhyaya, H. D., Bajaj, D., Gowda, C. L. L., Sharma, S., Tyagi, A. K., \& Parida, S. K. (2016). Identification of candidate genes and natural allelic variants for QTLs governing plant height in chickpea. Scientific Reports, 6, 27968.

Kujur, A., Upadhyaya, H. D., Shree, T., Bajaj, D., Das, S., Saxena, M. S., ... Parida, S. K. (2015). Ultra-high density intra-specific genetic linkage maps accelerate identification of functionally relevant molecular tags governing important agronomic traits in chickpea. Scientific Reports, 5, 9468.

Lipka, A. E., Tian, F., Wang, Q., Peiffer, J., Li, M., Bradbury, P. J., ... Zhang, Z. (2012). GAPIT: Genome association and prediction integrated tool. Bioinformatics, 2, 2397-2399.

Malik, N., Dwivedi, N., Singh, A. K., Parida, S. K., Agarwal, P., Thakur, J. K., \& Tyagi, A. K. (2016). An integrated genomic strategy delineates candidate mediator genes regulating grain size and weight in rice. Scientific Reports, 6, 23253.

Pandey, M. K., Roorkiwal, M., Singh, V. K., Ramalingam, A., Kudapa, H., Thudi, M., ... Varshney, R. K. (2016). Emerging genomic tools for legume breeding: Current status and future prospects. Frontiers in Plant Science, 7, 455.

Parween, S., Nawaz, K., Roy, R., Pole, A. K., Venkata Suresh, B., Misra, G., ... Chattopadhyay, D. (2015). An advanced draft genome assembly of a desi type chickpea (Cicer arietinum L.). Scientific Reports, 5, 12806.

Penmetsa, R. V., Carrasquilla-Garcia, N., Bergmann, E. M., Vance, L., Castro, B., Kassa, M. T., ... Cook, D. R. (2016). Multiple post-domestication origins of kabuli chickpea through allelic variation in a diversificationassociated transcription factor. New Phytologist, 211, 1440-1451.

Saxena, M. S., Bajaj, D., Das, S., Kujur, A., Kumar, V., Singh, M., ... Parida, S. K. (2014). An integrated genomic approach for rapid delineation of candidate genes regulating agro-morphological traits in chickpea. DNA Research, 21, 695-710.

Saxena, M. S., Bajaj, D., Kujur, A., Das, S., Badoni, S., Kumar, V., ... Parida, S. K. (2014). Natural allelic diversity, genetic structure and linkage disequilibrium pattern in wild chickpea. PLoS one, 9, e107484.

Saxena, S. C., Salvi, P., Kaur, H., Verma, P., Petla, B. P., Rao, V., ... Majee, M. (2013). Differentially expressed myo-inositol monophosphatase gene (CaIMP) in chickpea (Cicer arietinum L.) encodes a lithium sensitive phosphatase enzyme with broad substrate specificity and improves seed germination and seedling growth under abiotic stresses. Journal of Experimental Botany, 64, 5623-5639.

Toker, J. C. (2009). A note on the evolution of kabuli chickpeas as shown by induced mutations in Cicer reticulatum Ladizinsky. Genetic Resources and Crop Evolution, 56, 7-12.

Upadhyaya, H. D., Bajaj, D., Das, S., Kumar, V., Gowda, C. L. L., Sharma, S. ... Parida, S. K. (2016). Genetic dissection of seed-iron and zinc concentrations in chickpea. Scientific Reports, 6, 24050

Upadhyaya, H. D., Bajaj, D., Das, S., Saxena, M. S., Badoni, S., Kumar, V., ... Parida, S. K. (2015). A genome-scale integrated approach aids in genetic dissection of complex flowering time trait in chickpea. Plant Molecular Biology, 89, 403-420.

Van Ooijen, J. W. (2009). MapQTL 6: Software for the mapping of quantitative trait loci in experimental populations of diploid species. Netherlands: B.V. Kyazma, Wageningen.
Varshney, R. K., Mohan, S. M., Gaur, P. M., Gangarao, N. V. P. R., Pandey, M. K., Bohra, A., ... Gowda, C. L. L. (2013). Achievements and prospects of genomics-assisted breeding in three legume crops of the semi-arid tropics. Biotechnology Advances, 31, 1120-1134.

Varshney, R. K., Song, C., Saxena, R. K., Azam, S., Yu, S., Sharpe, A. G., ... Cook, D. R. (2013). Draft genome sequence of chickpea (Cicer arietinum) provides a resource for trait improvement. Nature Biotechnology, 31, 240-246.

Varshney, R. K., Thudi, M., Nayak, S. N., Gaur, P. M., Kashiwagi, J., Krishnamurthy, L., ... Viswanatha, K. P. (2014). Genetic dissection of drought tolerance in chickpea (Cicer arietinum L.). Theoretical Applied Genetics, 127, 445-462.

Voorrips, R. E. (2002). MapChart: Software for the graphical presentation of linkage maps and QTLs. Journal of Heredity, 93, 77-78.

Xia, Y., Ning, Z., Bai, G., Li, R., Yan, G., Siddique, K. H. M., ... Guo, P. (2012). Allelic variations of a light harvesting chlorophyll a/b-binding protein gene (Lhcb1) associated with agronomic traits in barley. PLoS one, 7, 1-9.

Xu, Y. H., Liu, R., Yan, L., Liu, Z. Q., Jiang, S. C., Shen, Y. Y., ... Zhang, D. P. (2012). Light-harvesting chlorophyll a/b-binding proteins are required for stomatal response to abscisic acid in Arabidopsis. Journal of Experimental Botany, 63, 1095-1106.

Yamori, W., Kondo, E., Sugiura, D., Terashima, I., Suzuki, Y., \& Makino, A (2016). Enhanced leaf photosynthesis as a target to increase grain yield: Insights from transgenic rice lines with variable rieske $\mathrm{FeS}$ protein content in the cytochrome $b_{6} / f$ complex. Plant Cell \& Environment, 39, 80-87.

Zhang, J., Du, H., Chao, M., Yin, Z., Yang, H., Li, Y., ... Yu, D. (2016). Identification of two $b Z I P$ transcription factors interacting with the promoter of soybean Rubisco activase gene (GmRCA $)$. Frontiers in Plant Science, 7, 1-14.

Zhang, Z., Ersoz, E., Lai, C. Q., Todhunter, R. J., Tiwari, H. K., Gore, M. A., .. Buckler, E. S. (2010). Mixed linear model approach adapted for genome-wide association studies. Nature Genetics, 42, 355-360.

Zhu, X. G., Ort, D. R., Whitmarsh, J., \& Long, S. P. (2004). The slow reversibility of photosystem II thermal energy dissipation on transfer from high to low light may cause large losses in carbon gain by crop canopies: A theoretical analysis. Journal of Experimental Botany, 55, 1167-1175.

\section{SUPPORTING INFORMATION}

Additional supporting information may be found online in the Supporting Information section at the end of the article.

How to cite this article: Basu U, Bajaj D, Sharma A, et al. Genetic dissection of photosynthetic efficiency traits for enhancing seed yield in chickpea. Plant Cell Environ. 2018;1-16. https://doi.org/10.1111/pce.13319 